\title{
Time of flight PET reconstruction using nonuniform update for regional recovery uniformity
}

\author{
Kyungsang Kim \\ Gordon Center for Medical Imaging, Massachusetts General Hospital and Harvard Medical School, 125 Nashua Street 6th floor, \\ Suite 660, Boston, MA02114, USA \\ Donghwan Kim \\ Department of Electrical Engineering and Computer Science, University of Michigan, Ann Arbor, MI48105, USA
}

Jaewon Yang

Physics Research Laboratory, Department of Radiology and Biomedical Imaging, University of California, San Francisco, CA94143, USA

\section{Georges El Fakhri}

Gordon Center for Medical Imaging, Massachusetts General Hospital and Harvard Medical School, 125 Nashua Street 6th floor, Suite 660, Boston, MA02114, USA

\section{Youngho Seo}

Physics Research Laboratory, Department of Radiology and Biomedical Imaging, University of California, San Francisco, CA94143, USA

Jeffrey A. Fessler

Department of Electrical Engineering and Computer Science, University of Michigan, Ann Arbor, MI48105, USA

\section{Quanzheng $\mathrm{Li}^{\mathrm{a})}$}

Gordon Center for Medical Imaging, Massachusetts General Hospital and Harvard Medical School, 125 Nashua Street 6th floor, Suite 660, Boston, MA02114, USA

(Received 16 March 2018; revised 19 November 2018; accepted for publication 20 November 2018; published 4 January 2019)

Purpose: Time of flight (TOF) PET reconstruction is well known to statistically improve the image quality compared to non-TOF PET. Although TOF PET can improve the overall signal to noise ratio (SNR) of the image compared to non-TOF PET, the SNR disparity between separate regions in the reconstructed image using TOF data becomes higher than that using non-TOF data. Using the conventional ordered subset expectation maximization (OS-EM) method, the SNR in the low activity regions becomes significantly lower than in the high activity regions due to the different photon statistics of TOF bins. A uniform recovery across different SNR regions is preferred if it can yield an overall good image quality within small number of iterations in practice. To allow more uniform recovery of regions, a spatially variant update is necessary for different SNR regions.

Methods: This paper focuses on designing a spatially variant step size and proposes a TOF-PET reconstruction method that uses a nonuniform separable quadratic surrogates (NUSQS) algorithm, providing a straightforward control of spatially variant step size. To control the noise, a spatially invariant quadratic regularization is incorporated, which by itself does not theoretically affect the recovery uniformity. The Nesterov's momentum method with ordered subsets (OS) is also used to accelerate the reconstruction speed. To evaluate the proposed method, an XCAT simulation phantom and clinical data from a pancreas cancer patient with full (ground truth) and $6 \times$ downsampled counts were used, where a Poisson thinning process was employed for downsampling. We selected tumor and cold regions of interest (ROIs) and compared the proposed method with the TOF-based conventional OS-EM and OS-SQS algorithms with an early stopping criterion.

Results: In computer simulation, without regularization, hot regions of OS-EM and OS-NUSQS converged similarly, but cold region of OS-EM was noisier than OS-NUSQS after 24 iterations. With regularization, although the overall speeds of OS-EM and OS-NUSQS were similar, recovery ratios of hot and cold regions reconstructed by the OS-NUSQS were more uniform compared to those of the conventional OS-SQS and OS-EM. The OS-NUSQS with Nesterov's momentum converged faster than others while preserving the uniform recovery. In the clinical example, we demonstrated that the OS-NUSQS with Nesterov's momentum provides more uniform recovery ratios of hot and cold ROIs compared to the OS-SQS and OS-EM. Although the cost function of all methods is equivalent, the proposed method has higher structural similarity (SSIM) values of hot and cold regions compared to other methods after 24 iterations. Furthermore, our computing time using graphics processing unit was $80 \times$ shorter than the time using quad-core CPUs. 
Conclusion: This paper proposes a TOF PET reconstruction method using the OS-NUSQS with Nesterov's momentum for uniform recovery of different SNR regions. In particular, the spatially nonuniform step size in the proposed method provides uniform recovery ratios of different SNR regions, and the Nesterov's momentum further accelerates overall convergence while preserving uniform recovery. The computer simulation and clinical example demonstrate that the proposed method converges uniformly across ROIs. In addition, tumor contrast and SSIM of the proposed method were higher than those of the conventional OS-EM and OS-SQS in early iterations. (C) 2018 American Association of Physicists in Medicine [https://doi.org/10.1002/mp.13321]

Key words: momentum, nonuniform update, NUSQS, recovery uniformity, TOF PET reconstruction

\section{INTRODUCTION}

Time of flight (TOF) positron emission tomography (PET) has been developed to improve image quality and reduce image acquisition time. ${ }^{1,2}$ The noise reduction has been studied using TOF and non-TOF data in many papers. ${ }^{3,4,5,6}$ One of the main advantages using TOF information is to improve the signal-to-noise ratio (SNR) of the reconstructed image. The SNR of an image voxel reconstructed by filtered backprojection (FBP) is approximately: ${ }^{7}$

$$
\mathrm{SNR}=k \cdot N^{-\frac{1}{2}}\left(\frac{T^{2}}{T+S+R}\right)^{\frac{1}{2}},
$$

where $k$ is a physically defined constant and $N$ is the number of voxels in an image. $T$ is the number of true counts in a back-projected image, $S$ and $R$ are the numbers of scatter and random counts, respectively, in a back-projected image. The noise equivalent count rate (NEC), defined by $T^{2} /(T+$ $S+R)$ with measurements, is also used as the effective sensitivity considering noise contributions, such as scatter and random events. ${ }^{8}$ For example, in a cylinder of diameter $D$ with uniform activity, the improvements of SNR and NEC gains of TOF PET are estimated as follows: ${ }^{8}$

$$
\mathrm{SNR}_{\mathrm{T}}=\sqrt{\frac{D}{\Delta x}} \mathrm{SNR}_{\mathrm{n}-\mathrm{T}}, \quad \mathrm{NEC}_{\mathrm{T}}=\frac{D}{\Delta x} \mathrm{NEC}_{\mathrm{n}-\mathrm{T}},
$$

where $\mathrm{T}$ and $\mathrm{n}-\mathrm{T}$ denote TOF and non-TOF, respectively, $\Delta x$ is the full-width-half-maximum (FWHM) calculated by an intrinsic time resolution $(\Delta t)$ as $\Delta x=c \Delta t / 2$. Here, $c$ is the speed of light. Therefore, TOF information improves the image quality with higher SNR and NEC. ${ }^{8}$ In addition, Karp et al. ${ }^{9}$ demonstrated that the contrast recovery coefficient (CRC) using TOF data converges faster than the CRC using non-TOF data in the maximum likelihood expectation maximization (MLEM) algorithm, and observed that CRCs using TOF data are larger than those using non-TOF data. Here, the CRC is defined as $(H-B) / B$, where $H$ is a hot region and $B$ is a background.

In general, due to nonuniform (NU) activity in an image, SNRs differ between regions. The standard iterative algorithms such as OS-EM ${ }^{10}$ and OS-SQS ${ }^{11}$ converge slowly for the low-SNR region, and regions with different SNRs converge to different recovery ratios. Although TOF PET can improve image contrast in high SNR regions, the disparity of
SNRs between elements in an image becomes considerably wider by at least $\sqrt{\frac{D}{\Delta x}}$ times compared to that in non-TOF PET. For example, specific regions, such as low activity regions, can be very noisy after a few iterations, potentially degrading the overall noise distribution within an image and making it difficult to terminate an iterative method early. Therefore, development of an algorithm that provides fast and uniform recovery of ROIs without sacrificing the overall convergence speed is necessary, which can help minimize the total number of iterations needed in practice.

In iterative image reconstruction, there are two possible ways to encourage the uniform recovery for different SNR regions: (a) spatially variant regularization based on the noise statistics of data and (b) spatially NU step size method for the gradientbased update. The spatially variant regularization has been used to encourage uniform image resolution. ${ }^{12,13,14}$ Based on our knowledge, there is no spatially variant regularization for achieving uniform recovery. Due to the complexity in developing a spatially variant regularization satisfying the requirement of uniform recovery, a spatially NU step size method is more straightforward to control the uniform recovery.

Algorithms using spatially variant step size have been developed to promote faster convergence by nonuniformly updating the image in iterations. ${ }^{15,16}$ Nonhomogeneous iterative coordinate descent (NH-ICD) was proposed to accelerate the conventional ICD algorithm. ${ }^{15}$ However, NH-ICD is difficult to parallelize because each voxel is updated sequentially. Van Slambrouck and Nuyts proposed a group-wise NU coordinate descent update for faster convergence speed, ${ }^{16}$ which is more amenable to parallelization than ICD-type methods, but is still limited by its group size. On the other hand, Kim et al. proposed a nonuniform separable quadratic surrogates (NUSQS) algorithm, ${ }^{17}$ in which the step size is spatially NU and the update is parallelizable, accelerating the convergence speed. The NU update in that work was used to accelerate $\mathrm{CT}$ reconstruction, whereas here we use a similar idea to encourage spatially uniform recovery ratio, and extend the NUSQS method for TOF PET reconstruction.

For additional acceleration, it is useful to combine the ordered subsets $(\mathrm{OS})^{10}$ and the Nesterov's momentum method ${ }^{18}$ that uses previous descent updates to provide momentum. Kim et al. exploited both OS and Nesterov's momentum for CT reconstruction, ${ }^{19}$ where the convergence speed using both the OS and momentum was significantly higher than speeds of conventional methods in early iterations. Similarly, we propose 
a TOF-PET reconstruction exploiting the ordered subsets nonuniform separable quadratic surrogates (OS-NUSQS) algorithm with the Nesterov's momentum method. To further reduce the computation time, we implemented the proposed method using a graphics processing unit (GPU), particularly forward and backward projectors for TOF reconstruction and the quadratic regularization.

To validate the proposed algorithm, we performed computer simulations using an XCAT phantom ${ }^{20}$ under various conditions, and compared the recovery ratios of ROIs and the reconstructed image qualitatively and quantitatively. We also performed an experiment with a patient having pancreatic and other metastatic tumors. The image reconstructed after 300 iterations of one-subset version of EM using full data is used as the ground truth, and reconstructed images using data with $6 \times$ downsampled counts are used for validating the performance. Here, a Poisson thinning process is used for downsampling the counts of prompt raw data. ${ }^{21}$ The proposed method is compared with the conventional OS-SQS and OS-EM methods after certain number of iterations. More specifically, reconstructed images are compared after 24 iterations. Note that the OS-SQS has a relatively uniform step size compared to the OS-EM and OS-NUSQS, and the OS-EM is the standard method in iterative PET reconstruction, having a spatially variant update in iteration. We select both tumor and cold ROIs, and compared recovery ratios of ROIs and structural similarity (SSIM) values. Our results demonstrate that the proposed method can achieve uniform recovery ratios of ROIs, and provides a good image quality after a finite number of iterations.

This paper is organized as follows. Section II gives the problem formulation and the proposed method: OS-NUSQS and Nesterov's momentum for TOF PET reconstruction. Section III provides experimental setup details of computer simulation, clinical example, evaluation, and GPU implementation. Section IV demonstrates simulation and clinical results for various aspects. Section V discusses several technical issues and Section VI concludes.

\section{THEORY}

\section{A. Problems}

We reconstruct a non-negative image $x=\left(x_{1}, \ldots\right.$, $\left.x_{N_{v}}\right) \in \mathbb{R}_{+}^{N_{v}}$ from a time of flight (TOF) measurement $Y=\left[y^{1}, y^{2}, \ldots, y^{t}, \ldots, y^{N_{T}}\right] \in \mathbb{Z}_{+}^{N_{m} \times N_{T}} ; y^{t}=\left(y_{1}^{t}, \ldots, y_{N_{m}}^{t}\right) \in$ $\mathbb{Z}_{+}^{N_{m}} . N_{v}, N_{m}$ and $N_{T}$ denote the numbers of voxels, sinogram bins and TOF time bins, respectively. $Y$ is the number of photon counts and contains true, scatter, and random coincidence events in which we assume a Poisson statistical model:

$$
y_{i}^{t} \sim \operatorname{Poisson}\left\{\left[A_{t} x\right]_{i}+r_{i}^{t}\right\},
$$

where $y_{i}^{t}$ is the number of counts with the $i$ th sinogram at the $t$ th time bin. $r_{i}^{t}$ is the mean value of scatter and random events, ${ }^{22}$ with the $i$ th sinogram at the $t$ th time bin. $A_{t}=\left(A \circ W_{t}\right)$ is the TOF system matrix at the $t$ th time bin; $A \in \mathbb{R}_{+}^{N_{m} \times N_{v}}$ is the conventional system matrix that computes the line integral of a line of response (LOR), $W_{t} \in \mathbb{R}_{+}^{N_{m} \times N_{v}}$ is the Gaussian kernel along all LORs calculated by the TOF time response function, and $\circ$ is the Hadamard product. The TOF time response function is a one-dimensional Gaussian function centered at the emission position. $^{23}$ Thus, $\left[A_{t} x\right]_{i}=\sum_{j=1}^{N_{v}} a_{i j} w_{i j}^{t} x_{j}$ represents the Gaussian weighted line integral of a LOR for the th time bin and $w$ is the Gaussian weight. Here, $a_{i j}$ denotes the probability that a pair of annihilation photons emitted from the $j$ th voxel is detected at the $i$ th sinogram bin, and $w_{i j}^{t}$ is the Gaussian weight along the line of flight at $t$ th time bin. Throughout the paper, we use $a_{i j}^{t}=a_{i j} w_{i j}^{t}$.

For regularized TOF PET image reconstruction, we minimize the following cost function $\Psi_{o}(x)$ :

$$
\begin{aligned}
& \Psi_{o}(x)=L(x)+R(x) \\
& =\sum_{t=1}^{N_{T}} \sum_{i=1}^{N_{m}} h_{i}^{t}\left(\left[A_{t} x\right]_{i}\right)+\sum_{j=1}^{N_{v}} \psi_{j}(x),
\end{aligned}
$$

where $L(x)$ denotes the negative log-likelihood function from the Poisson statistics and $R(x)$ is a quadratic roughness regularization. ${ }^{24} h_{i}^{t}(k)=k+r_{i}^{t}-y_{i}^{t} \log \left(k+r_{i}^{t}\right), \psi_{j}(x)=\frac{\beta}{2} \sum_{j^{\prime} \in \Omega_{j}}$ $\rho_{j j^{\prime}}\left(x_{j}-x_{j^{\prime}}\right)^{2}$ and $\beta>0$ is a regularization parameter that controls the noise variance of the reconstructed image. $\rho_{j j^{\prime}}$ is the reciprocal of Euclidean distance between voxels $j$ and $j^{\prime}$, and $\Omega_{j}$ is the neighbor of center voxel $j$. For $\Omega_{j}$, we use 26 neighbor voxels in a three-dimensional (3D) space.

\section{B. Nonuniform separable quadratic surrogates for TOF PET}

In TOF-PET reconstruction, because the negative loglikelihood function $L(x)$ is difficult to minimize directly, a separable quadratic surrogates (SQS) algorithm for solving $L(x)$ is widely used with a regularization for reducing noise. $^{11,25}$

First, the quadratic surrogate function of $L(x)$ is as follows:

$$
L(x) \leq Q_{L}^{(n)}(x) \triangleq \sum_{t=1}^{N_{T}} \sum_{i=1}^{N_{m}} p_{i}^{t,(n)}\left(\left[A_{t} x\right]_{i}\right),
$$

where

$$
\begin{aligned}
& p_{i}^{t,(n)}(k) \triangleq h_{i}^{t}\left(k_{i}^{t,(n)}\right)+\dot{h}_{i}^{t}\left(k_{i}^{t,(n)}\right)\left(k-k_{i}^{t,(n)}\right)+\frac{v_{i}^{t,(n)}}{2} \\
& \left(k-k_{i}^{t,(n)}\right)^{2} \text {. }
\end{aligned}
$$

$k_{i}^{t,(n)}=\left[A_{t} x^{(n)}\right]_{i}$ at $n$th iteration, and $v_{i}^{t,(n)}$ is the curvature of $p_{i}^{t,(n)}(k)$. The first and second derivatives of $h_{i}^{t}$ are as follows ${ }^{11}$ :

$$
\begin{aligned}
& \dot{h}_{i}^{t}\left(k_{i}^{t,(n)}\right)=1-\frac{y_{i}^{t}}{k_{i}^{t,(n)}+r_{i}^{t}}, \\
& \ddot{h}_{i}^{t}\left(k_{i}^{t,(n)}\right)=\frac{y_{i}^{t}}{\left(k_{i}^{t,(n)}+r_{i}^{t}\right)^{2}} .
\end{aligned}
$$


For $v_{i}^{t,(n)}$, the optimal curvature ${ }^{25}$ is

$$
\begin{aligned}
v_{i}^{t,(n)}\left(k^{t,(n)}\right)= & \left\{\begin{array}{l}
{\left[2 \frac{h_{i}^{t}(0)-h_{i}^{t}\left(k^{t,(n)}\right)+\dot{h}_{i}^{t}\left(t^{t,(n)}\right)\left(k^{t,(n)}\right)}{\left(k^{t,(n)}\right)^{2}}\right]_{+}, k^{t,(n)}>0} \\
{\left[\ddot{h_{i}^{t}}(0)\right]_{+}, k^{t,(n)}=0 .}
\end{array}\right.
\end{aligned}
$$

[ $]_{+}$denotes an operator that sets negative values to zero. To reduce the computing cost, we can use the Newton curvature $\ddot{h}_{i}^{t}\left(\left[A_{t} x^{(n)}\right]_{i}\right)$, or precompute as $1 / \max \left(y_{i}^{t}, \epsilon\right)$, or approximately compute as $1 / \max \left(\left[A_{t} x^{(n)}\right]_{i}, \epsilon\right.$ ) (see Section V). Here, many of TOF measurements are zero, thus we set $\varepsilon$ as a small positive value.

We next review a separable surrogate of the quadratic surrogate function, ${ }^{11}$ which uses the following trick:

$$
\begin{aligned}
{\left[A_{t} x\right]_{i} } & =\sum_{j=1}^{N_{v}} a_{i j}^{t} x_{j} \\
& =\sum_{j=1}^{N_{v}} g_{i j}^{t,(n)}\left(\frac{a_{i j}^{t}}{g_{i j}^{t,(n)}}\left(x_{j}-x_{j}^{(n)}\right)+\left[A_{t} x^{(n)}\right]_{i}\right),
\end{aligned}
$$

where $g_{i j}^{t}=a_{i j}^{t} /\left(\sum_{j^{\prime}=1}^{N_{v}} a_{i j^{\prime}}^{t}\right)$ is a non-negative real value $\left(g_{i j}^{t}=0\right.$ only if $a_{i j}^{t}=0$ for all $\left.i, j\right)$, and $\sum_{j=1}^{N_{v}} g_{i j}^{t,(n)}=1$. By using the convexity of $p_{i}^{t,(n)}$, the convexity inequality can be expressed as:

$$
p_{i}^{t,(n)}\left(\left[A_{t} x\right]_{i}\right) \leq \sum_{j=1}^{N_{v}} g_{i j}^{t,(n)} p_{i}^{t,(n)}\left(\frac{a_{i j}^{t}}{g_{i j}^{t,(n)}}\left(x_{j}-x_{j}^{(n)}\right)+\left[A_{t} x^{(n)}\right]_{i}\right) .
$$

Combined with Eq. (5), we have the following majorizer:

$$
L(x) \leq Q_{L}^{(n)}(x) \leq \phi_{L}^{(n)}(x) \triangleq \sum_{t=1}^{N_{T}} \sum_{j=1}^{N_{v}} \phi_{L, j}^{t,(n)}\left(x_{j}\right)
$$

where

$$
\phi_{L, j}^{t,(n)}\left(x_{j}\right) \triangleq \sum_{i=1}^{N_{m}} g_{i j}^{t,(n)} p_{i}^{t,(n)}\left(\frac{a_{i j}^{t}}{g_{i j}^{t,(n)}}\left(x_{j}-x_{j}^{(n)}\right)+\left[A_{t} x^{(n)}\right]_{i}\right) .
$$

The second derivative of the surrogate function $\phi_{L, j}^{(n)}\left(x_{j}\right)$ is

$$
d_{L, j}^{(n)} \triangleq \frac{\partial^{2}}{\partial x_{j}^{2}} \phi_{L, j}^{(n)}\left(x_{j}\right)=\sum_{t=1}^{N_{T}} \sum_{i=1}^{N_{m}} v_{i}^{t,(n)}\left(a_{i j}^{t}\right)^{2} / g_{i j}^{t} .
$$

We next derive a separable surrogate of the quadratic roughness regularization $R(x)::^{25}$

$$
\begin{gathered}
R(x)=\sum_{j=1}^{N_{v}} \psi_{j}(x)=\sum_{j=1}^{N_{v}} \frac{\beta}{2} \sum_{j^{\prime} \in \Omega_{j}} \rho_{j j^{\prime}}\left(x_{j}-x_{j^{\prime}}\right)^{2}, \\
=\sum_{j=1}^{N_{v}} \sum_{j^{\prime} \in \Omega_{j}} \frac{\beta \rho_{j j^{\prime}}}{2}\left(\frac{\left(2 x_{j}-x_{j}^{(n)}-x_{j^{\prime}}^{(n)}\right)}{2}+\frac{\left(-2 x_{j^{\prime}}+x_{j}^{(n)}+x_{j^{\prime}}^{(n)}\right)}{2}\right)^{2},
\end{gathered}
$$

$$
\begin{aligned}
& \leq \sum_{j=1}^{N_{v}} \sum_{j^{\prime} \in \Omega_{j}} \frac{\beta \rho_{j j^{\prime}}}{4}\left(\left(2 x_{j}-x_{j}^{(n)}-x_{j^{\prime}}^{(n)}\right)^{2}+\left(2 x_{j^{\prime}}-x_{j}^{(n)}-x_{j^{\prime}}^{(n)}\right)^{2}\right) \\
& =\sum_{j=1}^{N_{v}} \sum_{j^{\prime} \in \Omega_{j}} \frac{\beta \rho_{j j^{\prime}}}{2}\left(2 x_{j}-x_{j}^{(n)}-x_{j^{\prime}}^{(n)}\right)^{2} \\
& \triangleq \sum_{j=1}^{N_{v}} \phi_{R, j}^{(n)}\left(x_{j}\right)=\phi_{R}^{(n)}(x)
\end{aligned}
$$

here, we use the convexity inequality in (17) and symmetry of quadratic function $\left(\psi_{j}(k)=\psi_{j}(-k)\right)$ in (18). The regularizing factor between two voxels of $j$ and $j^{\prime}$ is computed twice when switching center and neighbor, thus we can simplify the equation for $x_{j}$ in (19). The first and second derivatives of $\phi_{R}^{(n)}(x)$ at the point $x^{(n)}$ are as follows:

$$
\begin{aligned}
& \dot{\phi}_{R, j}^{(n)}\left(x^{(n)}\right)=2 \beta \sum_{j^{\prime} \in \Omega_{j}} \rho_{j j^{\prime}}\left(x_{j}^{(n)}-x_{j^{\prime}}^{(n)}\right), \\
& \ddot{\phi}_{R, j}^{(n)}(x)=4 \beta \sum_{j^{\prime} \in \Omega_{j}} \rho_{j j^{\prime}}=d_{R, j} .
\end{aligned}
$$

Now, the majorizer $\Psi(x)$ is:

$$
\begin{aligned}
& \Psi_{o}(x) \leq \Psi(x)=\phi_{L}^{(n)}(x)+\phi_{R}^{(n)}(x), \\
& =\sum_{t=1}^{N_{T}} \sum_{j=1}^{N_{v}} \phi_{L, j}^{t,(n)}\left(x_{j}\right)+\sum_{j=1}^{N_{v}} \phi_{R, j}^{(n)}\left(x_{j}\right) .
\end{aligned}
$$

The SQS with quadratic regularization provides the voxelwise update at each iteration as follows:

$$
x_{j}^{(n+1)}=x_{j}^{(n)}-\frac{\dot{\phi}_{L, j}^{(n)}\left(x_{j}^{(n)}\right)+\dot{\phi}_{R, j}^{(n)}\left(x_{j}^{(n)}\right)}{d_{L, j}^{(n)}+d_{R, j}}, \quad \forall j \in\left[1, \ldots, N_{v}\right] .
$$

Then, the step size of SQS with quadratic regularization has this relationship: ${ }^{26}$

$$
\Delta_{j}^{(n)}=x_{j}^{(n+1)}-x_{j}^{(n)} \propto \frac{1}{d_{L, j}^{(n)}+d_{R, j}} .
$$

Because the $d_{R, j}$ does not change in iterations as shown in Eq. (22), the step size is mainly decided by $d_{L, j}^{(n)}$. To accelerate the SQS algorithm, a larger value of $g_{i j}^{t,(n)}$ (or equivalently smaller value of $\left.d_{L, j}^{(n)}\right)$ can encourage larger step size. Here, we choose $g_{i j}^{t,(n)}=\frac{a_{i j}^{t} u_{j}^{(n)}}{\sum_{j^{\prime}=1}^{N_{v}} a_{i^{\prime}}^{t} j_{j^{\prime}}^{(n)}}$ using the NU-based update factor $\left(u_{j}^{(n)}\right)$ as follows: $:^{17,27}$

$$
u_{j}^{(n)}=\max \left\{\left|x_{j}^{(n-1)}-x_{j}^{(n)}\right|, \delta\right\},
$$

where $\delta$ is a small positive value. Note that the $u_{j}^{(n)}$ is an approximation of the oracle NU factor $\left|x_{j}^{(n)}-x_{j}^{(\infty)}\right|$. Our 
experimental results show that applying a Gaussian filtering to noisy $u^{(n)}$ empirically provides a better approximation to the oracle NU factor. The Gaussian filtering does not affect the final image quality but affects the convergence speed at early iterations. The corresponding NU denominator is

$$
\hat{d}_{L, j}^{(n)}=\frac{1}{u_{j}^{(n)}} \sum_{t=1}^{N_{T}} \sum_{i=1}^{N_{m}} v_{i}^{t,(n)} a_{i j}^{t}\left(\sum_{j^{\prime}=1}^{N_{v}} a_{i j^{\prime}}^{t} u_{j^{\prime}}^{(n)}\right),
$$

which leads to the NU-based update relationship of $\Delta_{j}^{(n)} \propto u_{j}^{(n)}$ that encourages the voxel-wise NU step size. This is a key property that allows one to control the recovery ratio of each voxel by using NU step size; Section IV demonstrates numerically that a suitable NU step size can provide similar ROI recovery ratios across iterations in TOFPET reconstruction.

The NUSQS method exploits the surrogate function with a diagonal Hessian matrix $D^{(n)}$ of $\Psi(x) .{ }^{26}$ Specifically, the $j$ th diagonal element of $D^{(n)}$ is $D_{j}^{(n)}=\hat{d}_{L, j}^{(n)}+d_{R, j}$. Algorithm 1 presents the pseudo code for NUSQS (one-subset version).

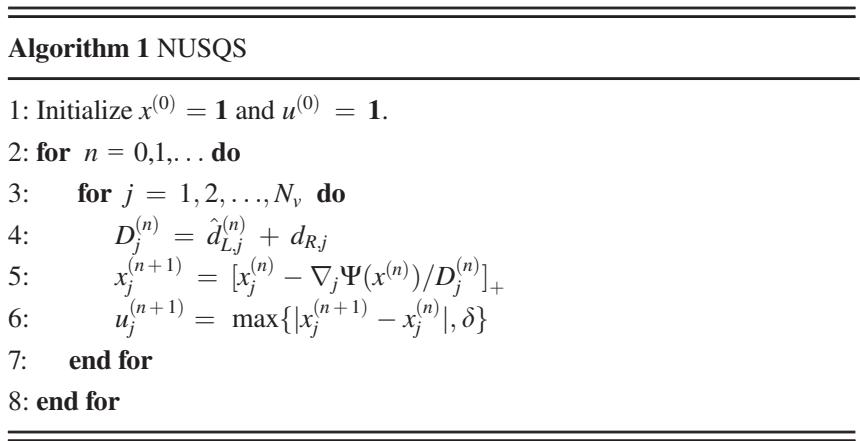

\section{C. Nesterov's momentum and ordered subsets methods}

To further accelerate the convergence speed, we exploit the Nesterov's momentum and OS methods. Iterative TOF-PET reconstruction requires the forward $A$ $\left(=\left[A_{1}, \ldots, A_{T}\right]\right)$ and backward $A^{H}\left(=\left[A_{1}^{H}, \ldots, A_{T}^{H}\right]\right)$ projection operators. We set $N_{S}$ as the number of subsets and the $s$ th-subset forward $A_{s} \quad\left(=\left[A_{s 1}, \ldots, A_{s T}\right]\right)$ and backward $A_{s}^{H}\left(=\left[A_{s 1}^{H}, \ldots, A_{s T}^{H}\right]\right)$ projection operators. Subsets are equally distributed over the angular bins. The computing cost per sub-iteration decreases almost linearly with the number of subsets $N_{s}$.

Now, the (approximate) majorizer with OS for TOF reconstruction is

$$
\Psi(x)=\sum_{s=1}^{N_{s}} \Psi_{s}(x),
$$

where

$$
\Psi_{s}(x)=\phi_{s L}^{(n)}(x)+\frac{1}{N_{s}} \phi_{R}^{(n)}(x),
$$

$$
\begin{aligned}
& =\sum_{t=1}^{N_{T}} \sum_{j=1}^{N_{v}} \phi_{s L, j}^{t,(n)}\left(x_{j}\right)+\frac{1}{N_{s}} \sum_{j=1}^{N_{v}} \phi_{R, j}^{(n)}\left(x_{j}\right), \\
& \phi_{s L, j}^{t,(n)}\left(x_{j}\right)=\sum_{i \in \Omega_{s}} g_{i j}^{t,(n)} p_{i}^{t,(n)}\left(\frac{a_{i j}^{t}}{g_{i j}^{t,(n)}}\left(x_{j}-x_{j}^{(n)}\right)+\left[A_{s t} x^{(n)}\right]_{i}\right), \\
& \hat{d}_{s L, j}^{t,(n)}=\frac{1}{u_{j}} \sum_{i \in \Omega_{s}} v_{i}^{t,(n)} a_{i j}^{t}\left(\sum_{j^{\prime}=1}^{N_{v}} a_{i j^{\prime}}^{t} u_{j^{\prime}}^{(n)}\right) .
\end{aligned}
$$

$\Omega_{s}$ denotes the sth subset and we evenly distribute the subsets in azimuthal bins. Algorithm 2 presents the pseudo code of OS-NUSQS. The subset balance can be approximately described as follows: ${ }^{10,19,26}$

$$
\begin{aligned}
& \nabla \Psi(x) \approx N_{s} \nabla \Psi_{s}(x), \\
& D^{(n)} \approx N_{s} D_{s}^{(n)},
\end{aligned}
$$

where $s=\left[1, \ldots, N_{s}\right]$ and $D_{s}$ is a diagonal Hessian of the surrogate function of $\Psi_{s}(x)$ for the sth subset; $D_{s j}^{(n)}$ is a $j$ th diagonal component of $D_{s}^{(n)}$.

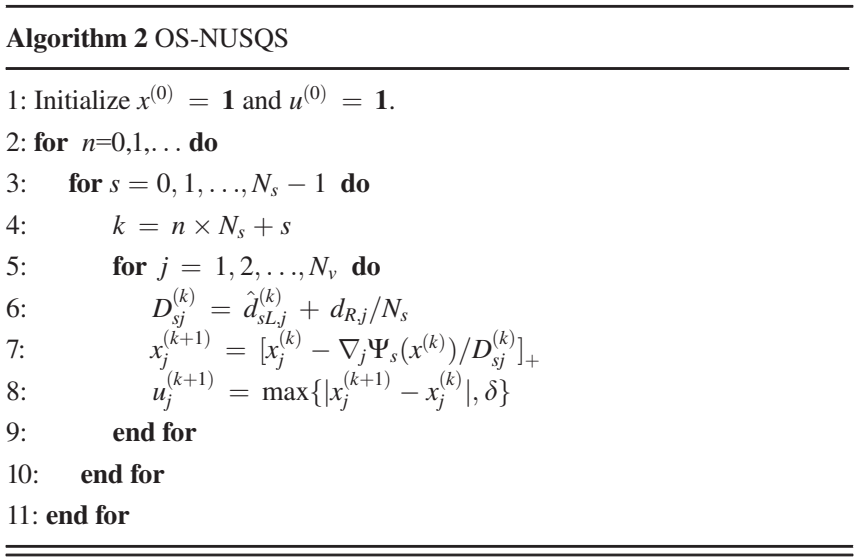

Next, we consider the OS-NUSQS combined with Nesterov's momentum. ${ }^{18,19}$ Specifically, the Nesterov's momentum $^{18}$ exploits the previous descent updates for additional acceleration. By combining with the OS method, we expect the convergence speed $O\left(1 /\left(n N_{S}\right)^{2}\right)$ in early iterations that is significantly faster than the speeds of NUSQS $O(1 / n)$ and OS-NUSQS $O\left(1 /\left(n N_{s}\right)\right)$. This is one of the main advantages of the OS in the momentum method, which accelerates approximately $\left(N_{s}\right)^{2}$ times in early iterations. ${ }^{19}$ Also, the momentum factor does not require additional memory because the NU factor $(u)$ can be reused in the momentum computation. In Algorithm 3, the NU factor $(u)$ is calculated in each iteration; to save computation, we first calculate the variation $x_{d}^{(k+1)}=\left(x^{(k+1)}-x^{(k)}\right)$ for momentum (line 10 in Algorithm 3), and then we update $u^{(k+1)}=\max \left\{\left|x_{d}^{(k+1)}\right|, \delta\right\}$ (line 11 in Algorithm 3). 


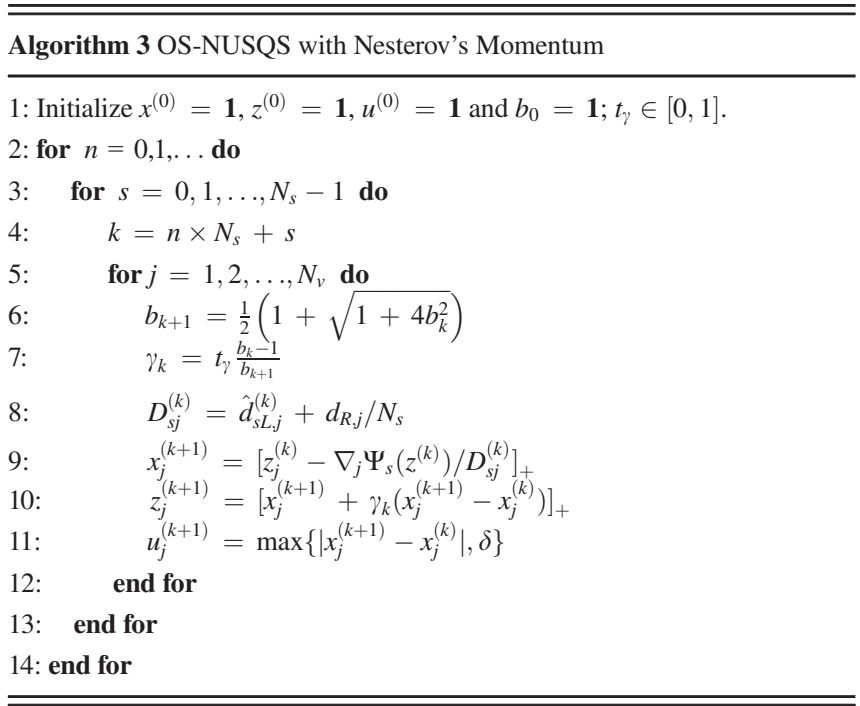

In addition, a relaxation factor $t_{\gamma}$ in Algorithm 3 is applied. Although the momentum method can practically improve the convergence speed, the OS-NUSQS with momentum algorithm can sometimes diverge due to $\mathrm{OS}^{19}{ }^{19}$ as will be presented in Section V. Therefore, we set the relaxation factor, such as $t_{\gamma} \in[0,1]$, to avoid divergence in the proposed method.

\section{EXPERIMENTAL SETUP}

\section{A. Computer simulation}

We performed a computer simulation using an XCAT phantom ${ }^{20}$ and the simulation geometry of a clinical Biograph mCT TOF PET/CT scanner (Siemens Medical Solutions USA, Inc., Knoxville, TN, USA). In a TOF sinogram, the number of radial bins with a $2.005 \mathrm{~mm}$ pixel size and azimuthal bins are 336, and the number of time bins is 13 with a $560 \mathrm{ps}$ time resolution; the radius of scanner is $427.6 \mathrm{~mm}$ and data were acquired with span 11 . In the reconstructed image, the number of image voxels is $336 \times 336 \times 109$ with a $2.005 \times 2.005 \times 2.027 \mathrm{~mm}^{3}$ voxel size. To evaluate ROIbased convergence, we chose three hot ROIs and one cold ROI at the lung, spine, and liver as shown in Fig. 1. In each region, a high-intensity component assumed as a tumor is added. Specifically, different tumor shapes and intensities were applied for evaluation of uniform recovery under different conditions. We put a sphere with a $7 \mathrm{~mm}$ radius and 0.4 intensity in the lung, a sphere with a $6 \mathrm{~mm}$ radius and 0.3 intensity in the spine, and an ellipsoid with axes of length $(15,7,7) \mathrm{mm}$ and 0.3 intensity in the liver as shown in Figs. 1(b)-1(d), respectively. The muscle (background) intensity is 0.02 . For ROI-based metric comparison, all ROIs were extracted by the shape of sphere with a $20 \mathrm{~mm}$ radius at the centers of tumors. The ground truths of tumor to muscle ratios (TMRs) are 20 in the lung, 15 in the spine, and 15 in the liver, respectively. We imposed Poisson noise to the prompt (attenuated) sinogram with signal to noise ratio (SNR) of $8 \mathrm{~dB}$ in which the number of counts was $3.3 \times 10^{7}$. In simulation, detector-pair sensitivities, scatter, and random counts were not considered.

We also conducted experiments to evaluate the recovery ratio. We used the same regularization $(\beta=0.2)$ for all methods. To consider not only tumor but also different background regions, we selected four sphere-shape ROIs with $20 \mathrm{~mm}$ radius, as shown in Fig. 1. Specifically, we considered the spine ROI with complex structures, the lung ROI with very low intensity, and the liver ROI with high-intensity backgrounds.

\section{B. Clinical example}

A pancreas-focused scan was performed for $45 \mathrm{~min}$ with a TOF PET/MR (SIGNA, GE Healthcare) scanner. A bolus injection of $196.1 \mathrm{MBq}$ of ${ }^{18} \mathrm{~F}-\mathrm{FDG}$ was administered. The protocol of this experiment was approved by the Institutional Review Board (IRB) of University of California, San Francisco (UCSF). The SIGNA scanner has $357 \times 224 \times$ $1981 \times 27$ (radial, angle, plane, TOF) bins with time of

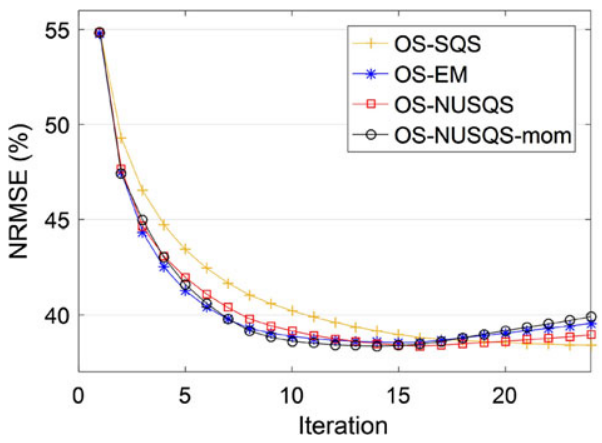

FIG. 2. Comparison of global NRMSEs for the OS-SQS, OS-EM, OSNUSQS, and OS-NUSQS with momentum. $\beta=0$ was used in simulation. [Color figure can be viewed at wileyonlinelibrary.com]

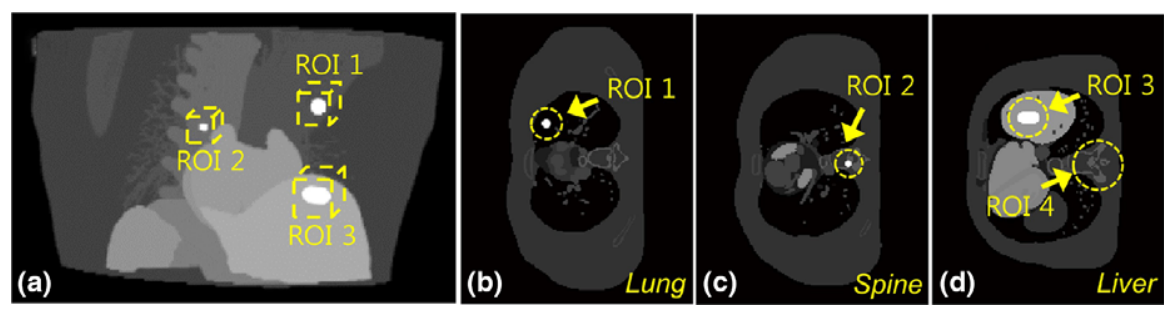

FIG. 1. XCAT phantom simulation setup using (a) three tumor ROIs with high-intensity components at the (b) lung, (c) spine, and (d) liver. A cold ROI in (d) was used for comparison. [Color figure can be viewed at wileyonlinelibrary.com] 

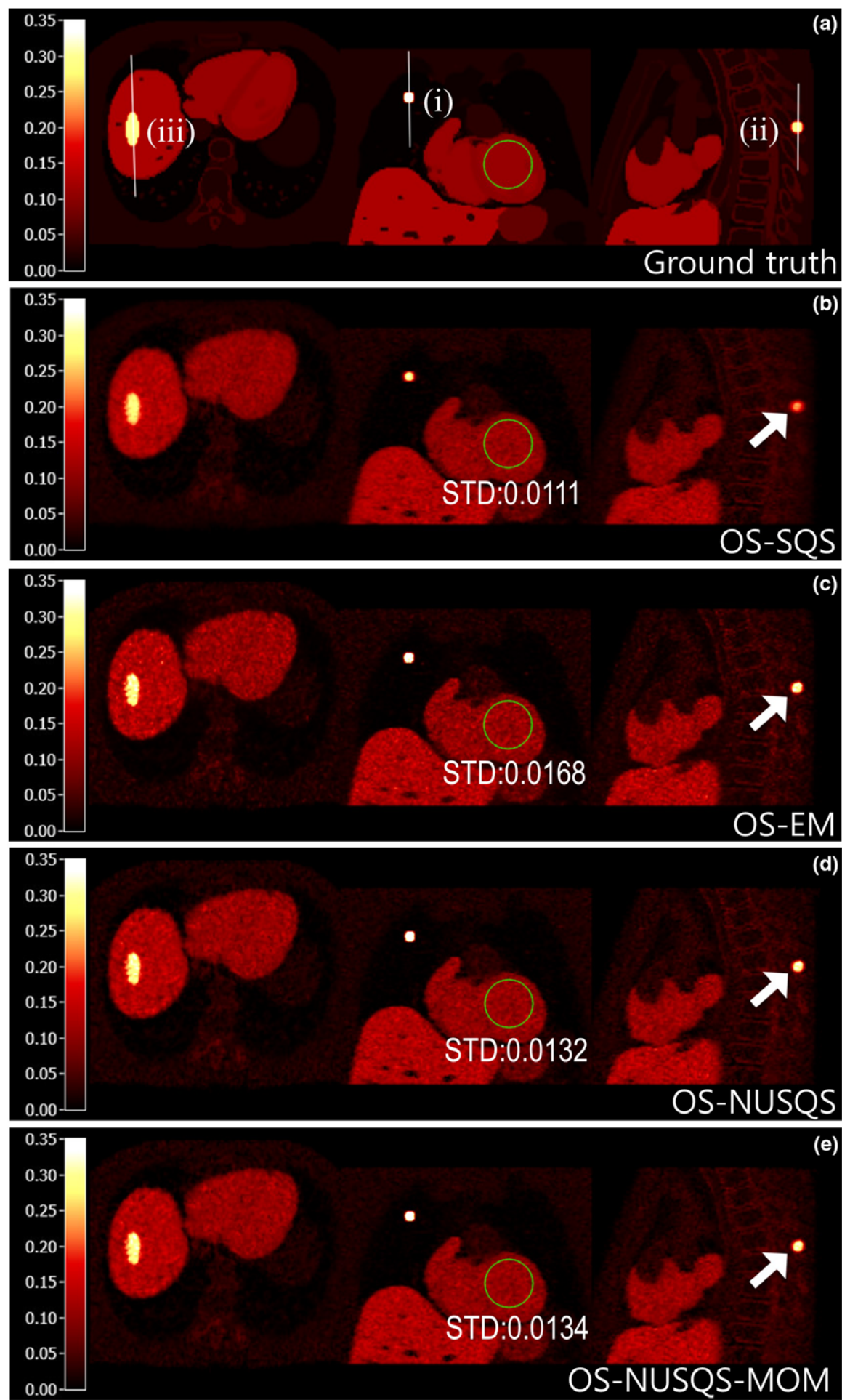

FIG. 3. (a) Ground truth and reconstructed images of (b) OS-SQS, (c) OS-EM, (d) OS-NUSQS, and (e) OS-NUSQS with momentum at 24 iterations. Standard deviations for a flat region of interest in coronal view were compared. $\beta=0$ was used in simulation. [Color figure can be viewed at wileyonlinelibrary.com]

flight resolution of $420 \mathrm{ps}$. The scanner radius is $640 \mathrm{~mm}$ and the field-of-view (FOV) is $600 \mathrm{~mm}$. The reconstructed image size was $256 \times 256 \times 89$ with $2.34 \times 2.34 \times$ $2.78 \mathrm{~mm}^{3}$ resolution.

For evaluation, we used the full-dose image as the ground truth and $6 \times$ downsampled data as a measurement. The full-dose image was acquired by EM (one-subset version of OS-EM) after 300 iterations. To generate $6 \times$ downsampled sinograms, a Poisson thinning process was used ${ }^{21}$ in which coincidence events can be randomly discarded by a predetermined sampling factor. The Poisson thinning process has been applied to initial prompt data (listmode or sinogram) 

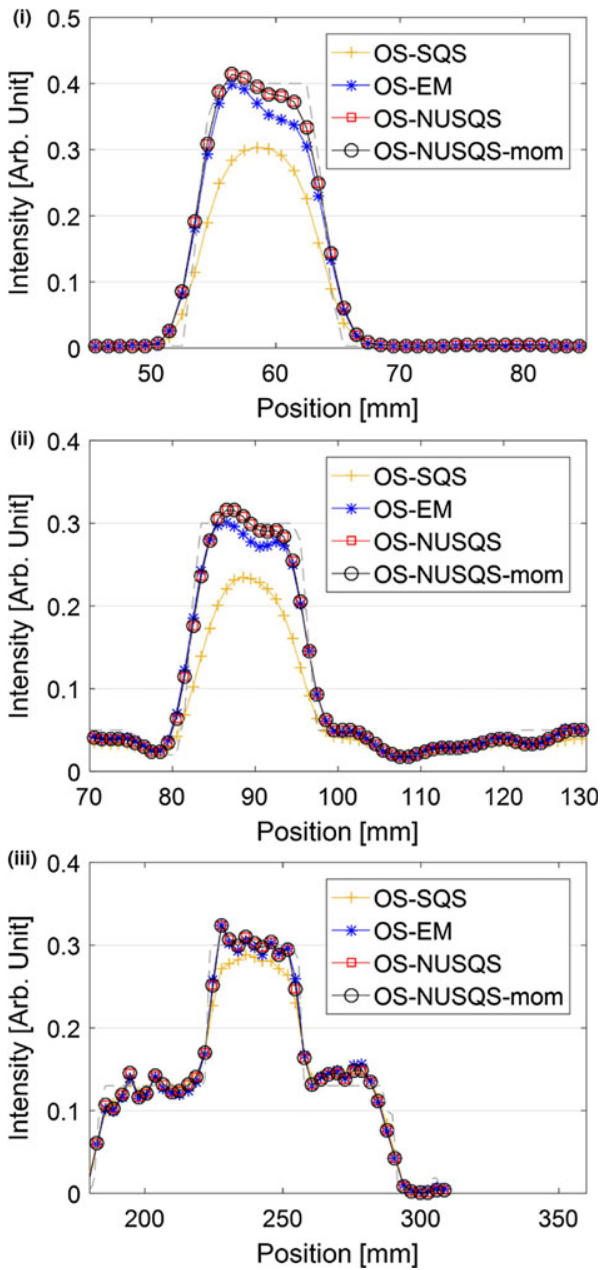

FIG. 4. Profiles of tumor ROIs in the (i) lung, (ii) spine, and (iii) liver as described in Fig. 3(a). Dot line is the ground truth. Profiles were measured with $\beta=0$ at 24 iterations in simulation. [Color figure can be viewed at wile yonlinelibrary.com]

before random, scatter, normalization, and attenuation corrections. We selected three tumor and one cold ROIs extracted by a sphere with a $12.5 \mathrm{~mm}$ radius. In patient data, detectorpair sensitivities (normalization), scatter, and random counts were fully considered.

\section{C. Evaluation}

In this paper, we compared the OS-NUSQS with the conventional OS-EM and OS-SQS. The step size of OS-SQS does not consider spatially different SNRs nor recovery variations. The OS-EM has a spatially variant step size; however, the step size of OS-EM does not take into account the recovery variation. We compared algorithms without regularization $(\beta=0)$ and with regularization $(\beta>0)$. For the regularized OS-EM algorithm, a De Pierro's EM algorithm ${ }^{28}$ is used for comparison, in which the quadratic regularization was equivalently used as in other methods. Throughout this paper, the iteration in result plots denotes the sub-iteration $(k)$, and we used eight subsets in reconstruction.

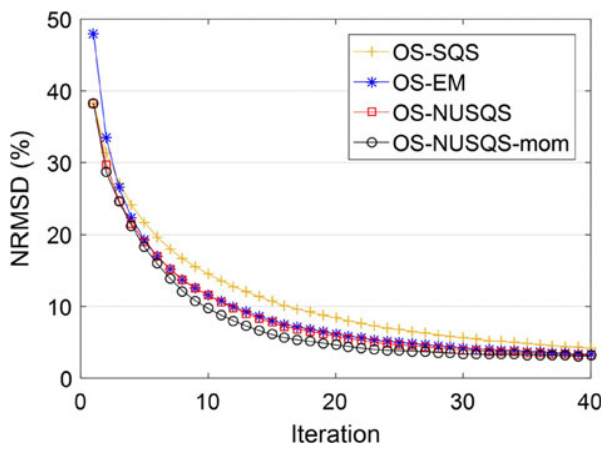

FIG. 5. Comparison of NRMSDs for OS-SQS, OS-EM, OS-NUSQS, and OS-NUSQS with momentum algorithms with quadratic regularization. $\beta=0.2$ was used in simulation. [Color figure can be viewed at wileyonline library.com]

The normalized root mean square error (NRMSE) $\sqrt{\sum_{j=1}^{N_{v}}\left(x_{j}^{*}-x_{j}^{(n)}\right)^{2}}$ $\sqrt{\sum_{j=1}^{N_{v}}\left(x_{j}^{*}\right)^{2}}$ was computed using the ground truth image $\left(x^{*}\right)$. To compare convergences of ROIs, we also calculated the ROI-based normalized root mean square difference (NRMSD) as $\frac{\sqrt{\sum_{j \in \Omega_{r}}\left(x_{j}^{(\infty)}-x_{j}^{(n)}\right)^{2}}}{\sqrt{\sum_{j \in \Omega_{r}}\left(x_{j}^{(\infty)}\right)^{2}}}$, where $n$ is the iteration, $x^{(\infty)}$ is the converged image after 300 iterations with one-subset version of OS-EM, and $\Omega_{r}$ is the $r$ th ROI. The recovery ratio was measured by $\frac{\sqrt{\sum_{j \in \Omega_{r}}\left(x_{j}^{*}-x_{j}^{(\infty)}\right)^{2}}}{\sqrt{\sum_{j \in \Omega_{r}}\left(x_{j}^{*}-x_{j}^{(n)}\right)^{2}}}$. Here, the distance between the ground truth and the converged image $\left(x^{*}-x^{(\infty)}\right)$ was used for calculating the recovery ratio (i.e., the recovery ratio approaches 1 with increasing iterations). Furthermore, we measure the ROI-based structural similarity (SSIM) index for comparing image quality. The SSIM is defined by $\frac{\left(2 \mu_{\mu^{*}} \mu_{r}+c_{1}\right)\left(2 \sigma_{r^{*}}+c_{2}\right)}{\left(\mu_{r^{*}}^{*}+\mu_{r}^{2}+c_{1}\right)\left(\sigma_{r^{*}}^{2}+\sigma_{r}^{2}+c_{2}\right)}$, where $r^{*}$ and $r$ denote the ROIs of the ground truth and the reconstructed image, respectively. $\mu$ is the average, $\sigma^{2}$ is the variance, and $\sigma_{r^{*} r}$ is the covariance of intensity within the ROIs $r^{*}$ and $r . c_{1}=2.5 \times 10^{-5}$ and $c_{2}=2.25 \times 10^{-4}$ were used.

\section{D. Implementation}

Parallel computing technologies are commonly used to speed up computations. In particular, the general purpose graphics processing unit (GPGPU) has been widely used for medical applications, such as 3D CT and PET reconstructions. ${ }^{29,30,31,32}$ To speed up the proposed method, we implemented TOF reconstruction using the GPU and compute unified device architecture (CUDA), similar to previous work. ${ }^{33}$ Specifically, we implemented our TOF system model with ray-driven forward projector and a matched (transpose) back projector with a time response function that is a typical Gaussian function with FWHM based on the timing 

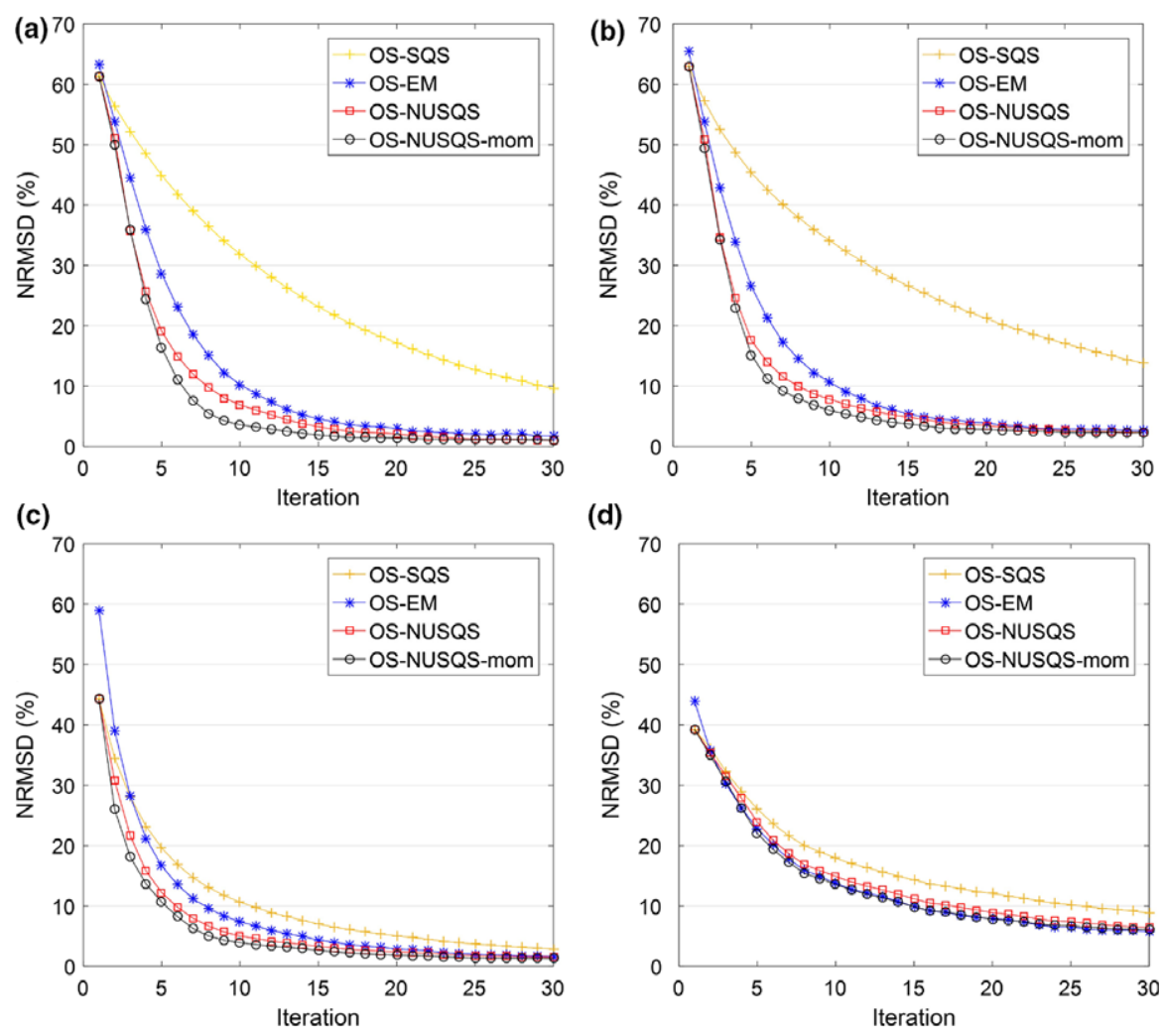

FIG. 6. Comparison of NRMSDs of OS-SQS, OS-EM, OS-NUSQS, and OS-NUSQS with momentum algorithms using quadratic regularizations for tumor ROIs in the (a) lung, (b) spine, (c) liver, and (d) cold region as shown in Fig. 1. $\beta=0.2$ was used in simulation. [Color figure can be viewed at wileyonlinelibra ry.com]

TABLE I. Tumor to muscle ratios of (a) OS-SQS, (b) OS-EM, (c) OS-NUSQS, and (d) OS-NUSQS with momentum. $\beta=0.2$ was used in simulation.

\begin{tabular}{lcccc}
\hline \hline TMR & OS-SQS & OS-EM & OS-NUSQS & OS-NUSQS-mom \\
\hline Lung & 13.7 & 14.4 & 14.5 & 14.5 \\
Spine & 10.7 & 11.7 & 11.8 & 11.8 \\
Liver & 12.7 & 12.7 & 12.8 & 12.8 \\
\hline \hline
\end{tabular}

resolution. In GPU kernels of TOF forward and backward projectors, each thread corresponds to a line-of-response (LOR); thus, all time bins are updated or used in each thread. Technically, we use the atomic operator to avoid interference from other threads when accessing a specific address at the same time, and the 3D linear interpolation using the texture memory is exploited for line integrals. A Gaussian coefficient table for the time response function is precalculated before the reconstruction process, and is assigned to the constant memory. All geometrical parameters are also assigned to the constant memory. The surrogate function of quadratic roughness regularization is easily parallelizable because the calculation of each voxel is independent, thus each thread in a GPU kernel can be assigned to each voxel. One main difference compared to the conventional PET reconstruction is that the denominator $D$ in algorithms $[1,2,3]$ cannot be precomputed due to the NU factor. Thus, the proposed method requires two forward and backward projections in each iteration. To further reduce the computing cost, our code calculates $A x^{(n)}$ and $A u^{(n)}$ at the same time in forward projection of GPU kernel that can share all geometrical computations; and the $\nabla \Psi(x)$ and $D$ are also calculated at the same time and share the geometrical computations in backward projection of GPU kernel as similarly done by Kim et al. ${ }^{19}$ This approach was $1.5 \sim 1.7$ times faster than separate calculations.

\section{RESULTS}

\section{A. Computer simulation}

\section{A.1. No regularization $(\beta=0)$}

First, to observe the convergence speed of different algorithms, we performed experiments without regularization, as a special case of $\beta=0$ in Eq. (5). The convergence speed of OS-NUSQS and OS-EM were similar as shown in Fig. 2. However, the OS-EM diverged faster than the OS-NUSQS. The OS-SQS at 24 iterations did not reach the minimum NRMSE. Figure 3 shows the reconstructed images of OSSQS, OS-EM, OS-NUSQS, and OS-NUSQS with momentum methods at 24 iterations. The OS-EM image was noisier than other images, particularly in the lower intensity region. We compared the standard deviations in the flat region with low intensity, a circle in coronal view as shown in Fig. 3. The 

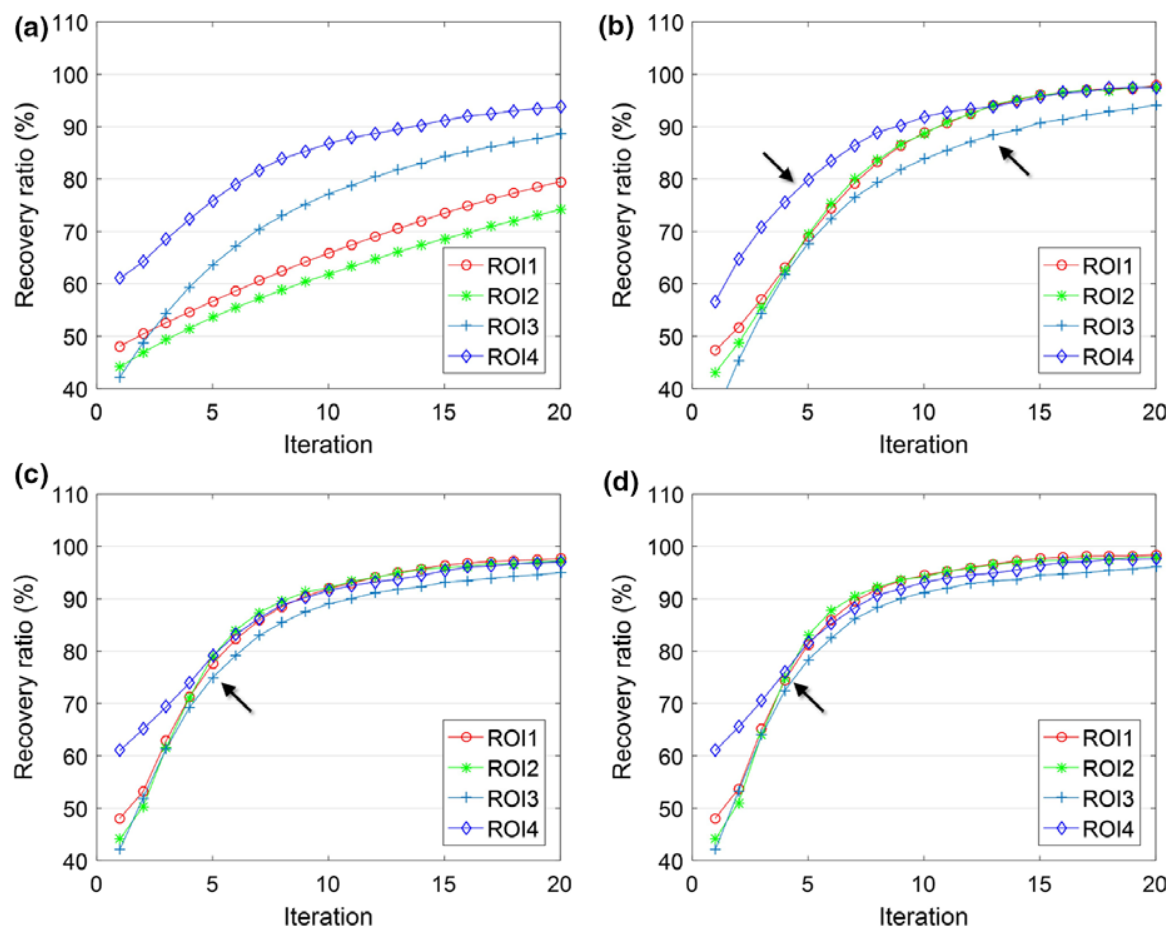

FIG. 7. ROI-based recovery ratio comparisons of (a) OS-SQS, (b) OS-EM, (c) OS-NUSQS, and (d) OS-NUSQS with momentum. $\beta=0.2$ was used in simulation. [Color figure can be viewed at wileyonlinelibrary.com]

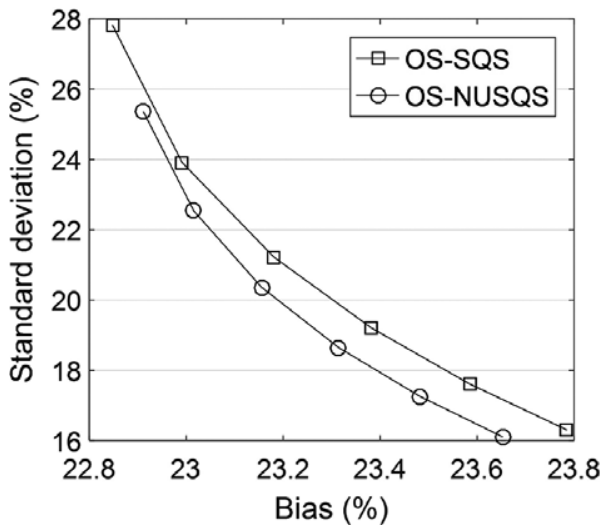

FIG. 8. Bias and standard deviation plots of OS-SQS and OS-NUSQS methods at 24 iterations. $\beta$ parameters between 0.08 and 0.3 were used in simulation. standard deviations of the flat region were 0.0111, 0.0168, 0.0132 , and 0.0134 for the OS-SQS, OS-EM, OS-NUSQS, and OS-NUSQS with momentum methods, respectively. The reconstructed images of OS-NUSQS with and without momentum were visually similar to the image of OS-EM, and we confirmed that OS-NUSQS has reduced noise compared to OS-EM in early iterations of TOF reconstruction. The OSSQS method did not reach convergence at 24 iterations, particularly at high-intensity regions, such as tumors in the lung and spine as shown in Fig. 3(b). For high-intensity regions, Fig. 4 compares profiles of tumors in the liver, lung, and spine regions as shown in Fig. 3(a). In Fig. 4, we observed that the profiles of OS-SQS did not reach convergence after 24 iterations, and demonstrated that the contrast of OSNUSQS was higher than that of OS-EM at 24 iterations. Although the intensity of OS-EM in the liver ROI was similar
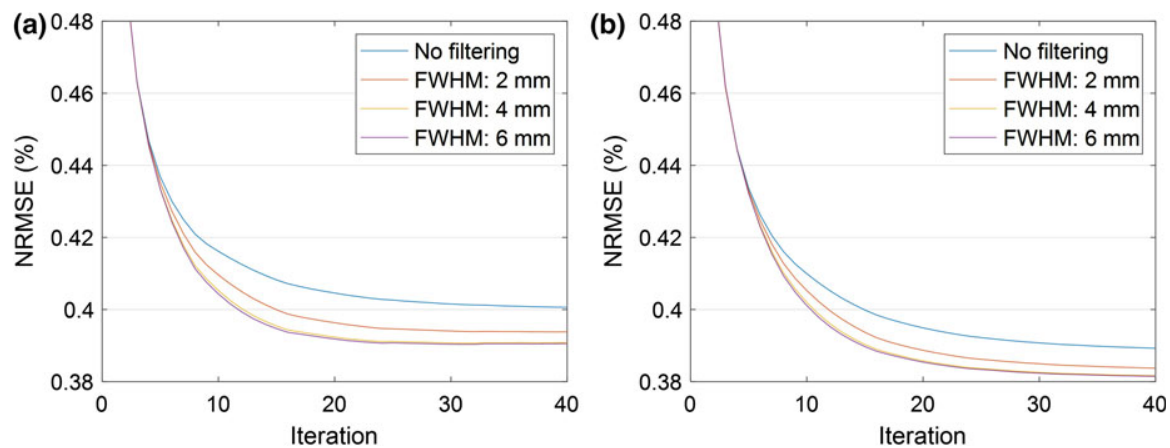

FIG. 9. NRMSE comparison without and with Gaussian filtering of FWHM 2, 4, $6 \mathrm{~mm}$ for different noise levels with total counts of (a) $1.98 \times 10^{7}$ and (b) $4.62 \times 10^{7} . \beta=0.2$ was used in simulation. [Color figure can be viewed at wileyonlinelibrary.com] 
to that of NUSQS, OS-EM needed more iterations for ROIs in the lung and spine. Note that profiles of OS-NUSQS with and without momentum were almost identical.

\section{A.2 With regularization $(\beta>0)$}

Figure 5 compares the NRMSDs for whole images of OSSQS, OS-EM, OS-NUSQS, and OS-NUSQS with momentum. The per-iteration convergence speed of OS-SQS was the slowest, and that of OS-NUSQS with momentum $\left(t_{\gamma}=0.7\right)$ was the fastest. In Fig. 6, the NRMSDs of tumor ROIs with OS-NUSQS converged much faster than those of OS-EM.
We compared the TMRs of OS-SQS, OS-EM, OS-NUSQS, and OS-NUSQS with momentum after 24 iterations in Table I. The TMRs of the proposed method were slightly higher than those of OS-SQS and OS-EM.

Figure 7 compares the recovery ratios of OS-SQS, OSEM, OS-NUSQS, and OS-NUSQS with momentum for four ROIs. In general, similar ratios of ROIs illustrate the uniform recovery of ROIs. We observed that the OS-NUSQS and OSNUSQS with momentum methods show uniform recovery ratios after five iterations for both hot and cold ROIs. In Fig. 8, the bias and standard deviation plots of OS-SQS and OS-NUSQS methods at 24 iterations were compared using
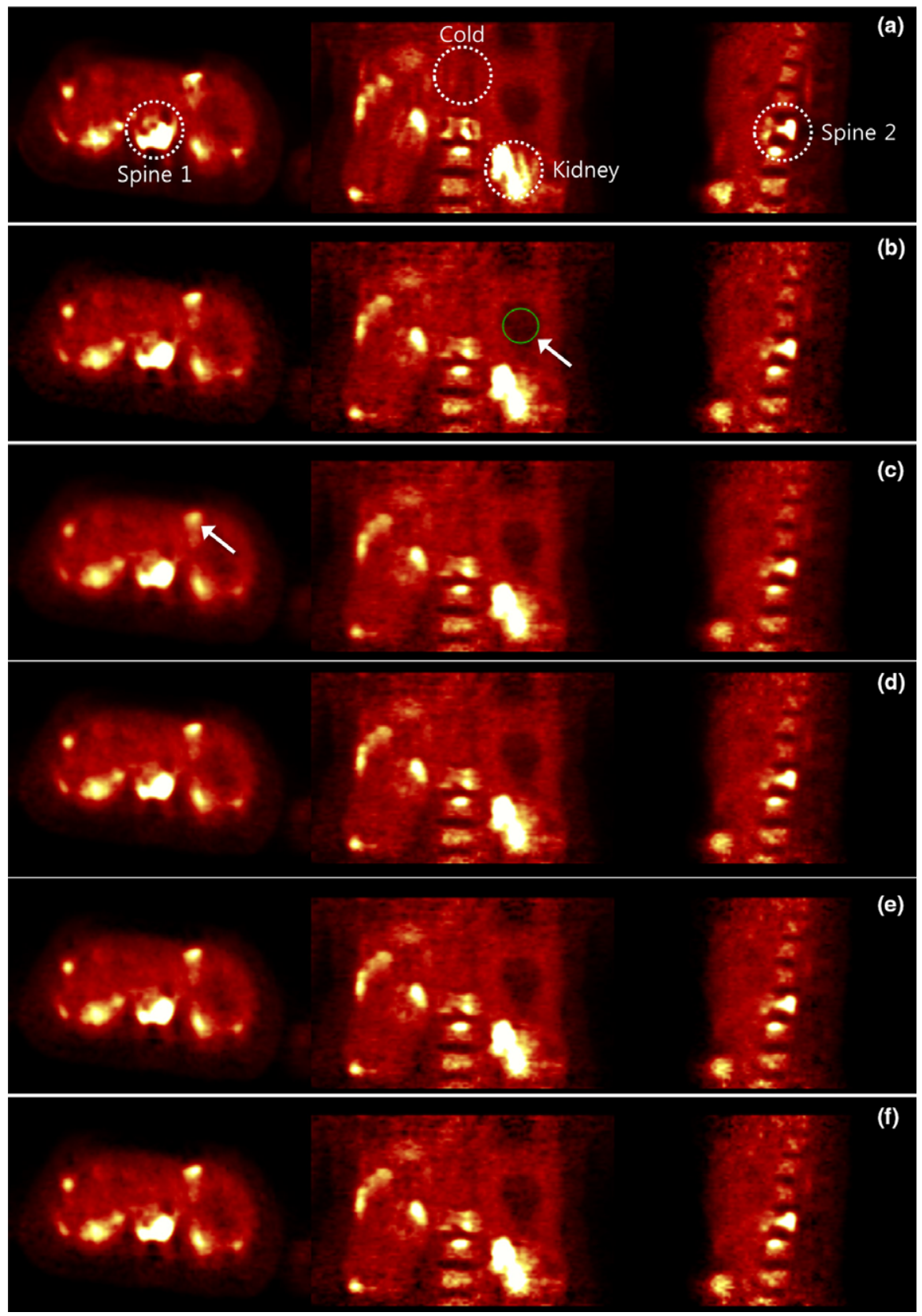

FIG. 10. (a) Ground truth of full-dose EM image, and reconstructed images of (b) converged OS-EM image with 300 iterations, (c) OS-SQS, (d) OS-EM, (e) OS-NUSQS, and (f) OS-NUSQS with momentum. (b)-(f) used $6 \times$ downsampled data. Three hot ROIs (spine 1 , kidney, and spine 2 ) were extracted at centers of tumors and a cold region was also extracted. $\beta=0.03$ was used with patient data. [Color figure can be viewed at wileyonlinelibrary.com] 
10 simulations (same SNR) with random Poisson noise. Figure 8 used $\beta$ parameters between 0.08 to 0.3 for both OSSQS and OS-NUSQS, which demonstrates that the NU method can improve the image quantitatively in early iterations. Here, the bias and standard deviation values of OSEM, OS-NUSQS, and OS-NUSQS with momentum were almost the same after 24 iterations. Note that after
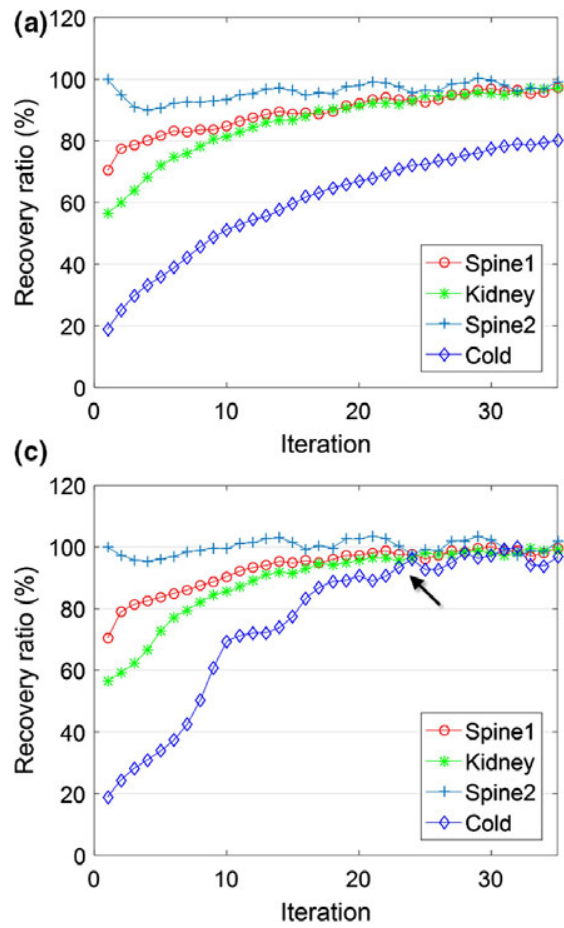

(d)
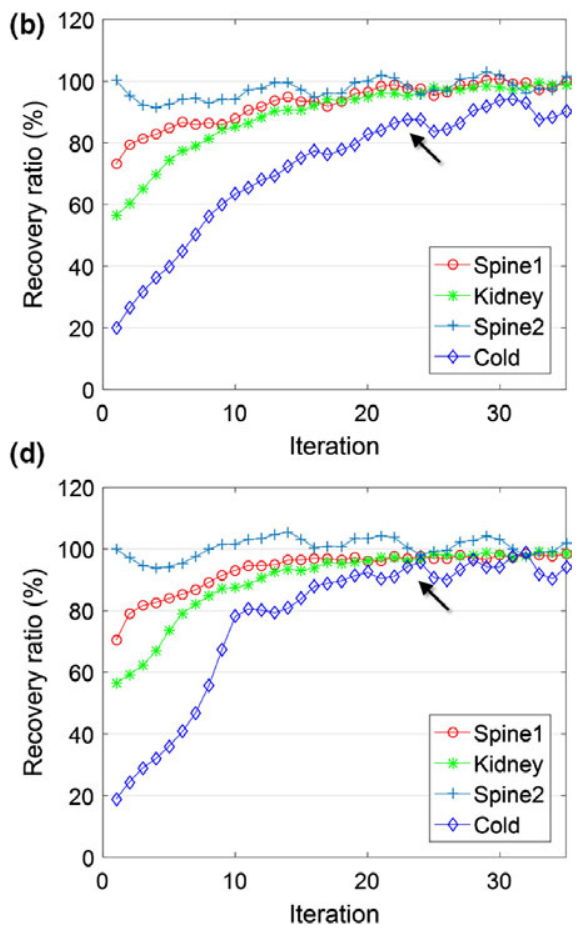

FIG. 11. ROI-based recovery ratio comparisons of (a) OS-SQS, (b) OS-EM, (c) OS-NUSQS, and (d) OS-NUSQS with momentum. $\beta=0.03$ was used with patient data. [Color figure can be viewed at wileyonlinelibrary.com]
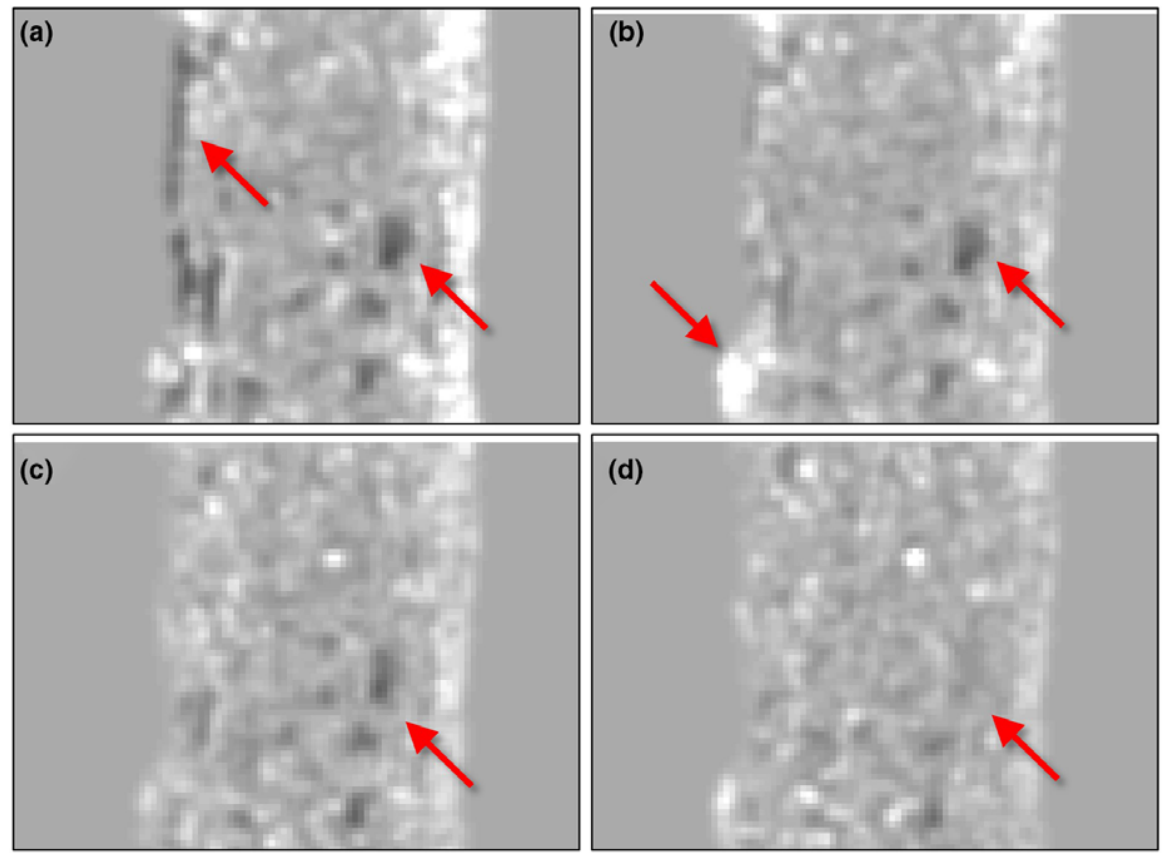

FIG. 12. Comparison of recovery images of (a) OS-SQS, (b) OS-EM, (c) OS-NUSQS, and (d) OS-NUSQS with momentum at 24 iterations. The saggital view of Fig. 10 was used. The intensity window is $[0,1.5]$. [Color figure can be viewed at wileyonlinelibrary.com] 
provide a better approximation of the oracle NU factor in our experiment, which lead to higher convergence speed at early iterations. To observe the relationship between noise levels and the optimal FWHM of Gaussian filtering, we compared the NRMSEs of reconstructed images using the NU factors without and with Gaussian filtering with FWHM of 2, 4, and $6 \mathrm{~mm}$, as shown in Fig. 9. Two noise levels with total counts of (a) $1.98 \times 10^{7}$ and $4.62 \times 10^{7}$ were used. Note that the total photon counts used in the simulation was $3.3 \times 10^{7}$. The NU factor using the Gaussian filtering showed fast decrease of NRMSEs at early iterations. The performance with Gaussian filtering of FWHM larger than $4 \mathrm{~mm}$ was almost the same. We observed that the FWHM of Gaussian filtering was not highly related to the noise level; thus, an FWHM of $4 \mathrm{~mm}$ was used for the Gaussian filtering in NU-based algorithms.

\section{B. Clinical example}

To evaluate the recovery ratio with converged images, we performed reconstructions with quadratic regularization

TABLE II. ROI-based SSIM comparisons of OS-SQS, OS-EM, OS-NUSQS, and OS-NUSQS with momentum. $\beta=0.03$ was used with patient data.

\begin{tabular}{lcccc}
\hline \hline SSIM & OS-SQS & OS-EM & OS-NUSQS & OS-NUSQS-mom \\
\hline Spine 1 & 0.841 & 0.850 & $\mathbf{0 . 8 5 2}$ & 0.840 \\
Kidney & 0.727 & 0.741 & $\mathbf{0 . 7 4 8}$ & 0.724 \\
Spine 2 & 0.688 & 0.690 & $\mathbf{0 . 6 9 4}$ & 0.683 \\
Cold & 0.942 & 0.953 & 0.963 & $\mathbf{0 . 9 8 2}$ \\
\hline \hline
\end{tabular}

The bold values represent the highest SSIM metrics. having $\beta=0.03$ and relaxation factor of 0.5 for the Nesterov's momentum. We will additionally discuss effects of parameters such as the number of subsets and relaxation factor in Section V.

Figure 10 shows the reconstructed images of OS-SQS, OS-EM, OS-NUSQS, and OS-NUSQS with momentum methods at 24 iterations. Figure 10(a) is the ground truth of full-dose EM image after 300 iterations, and Fig. 10(b) is the converged EM image of $6 \times$ downsampled data after 300 iterations. The OS-SQS was not fully converged in Fig. 10(c) (see arrow), however, other methods converged after 24 iterations.

Figure 11 compares the recovery ratios of OS-SQS, OSEM, OS-NUSQS, and OS-NUSQS with momentum for four ROIs. The OS-NUSQS and OS-NUSQS with momentum methods show uniform recovery ratios after 24 iterations for both hot and cold ROIs. In Fig. 12, we also compared the recovery images of OS-SQS, OS-EM, OS-NUSQS, and OSNUSQS with momentum, where the voxel-wise recovery ratio was calculated at 24 iterations and the saggital view in Fig. 10 was used. Because of the high noise of recovery image, a Gaussian filtering with FWHM $2.5 \mathrm{~mm}$ was additionally applied only for visualization in Fig. 12. Boundaries and inner regions in the recovery images of OS-SQS and OSEM were not uniform at 24 iterations. Although the recovery ratios of specific ROIs of OS-NUSQS and OS-NUSQS with momentum were similar, the recovery image of OS-NUSQS with momentum was more uniform than the recovery image of OS-NUSQS.

For image quality comparison, we compared ROI-based SSIM values as shown in Table II. The SSIMs of NUSQS
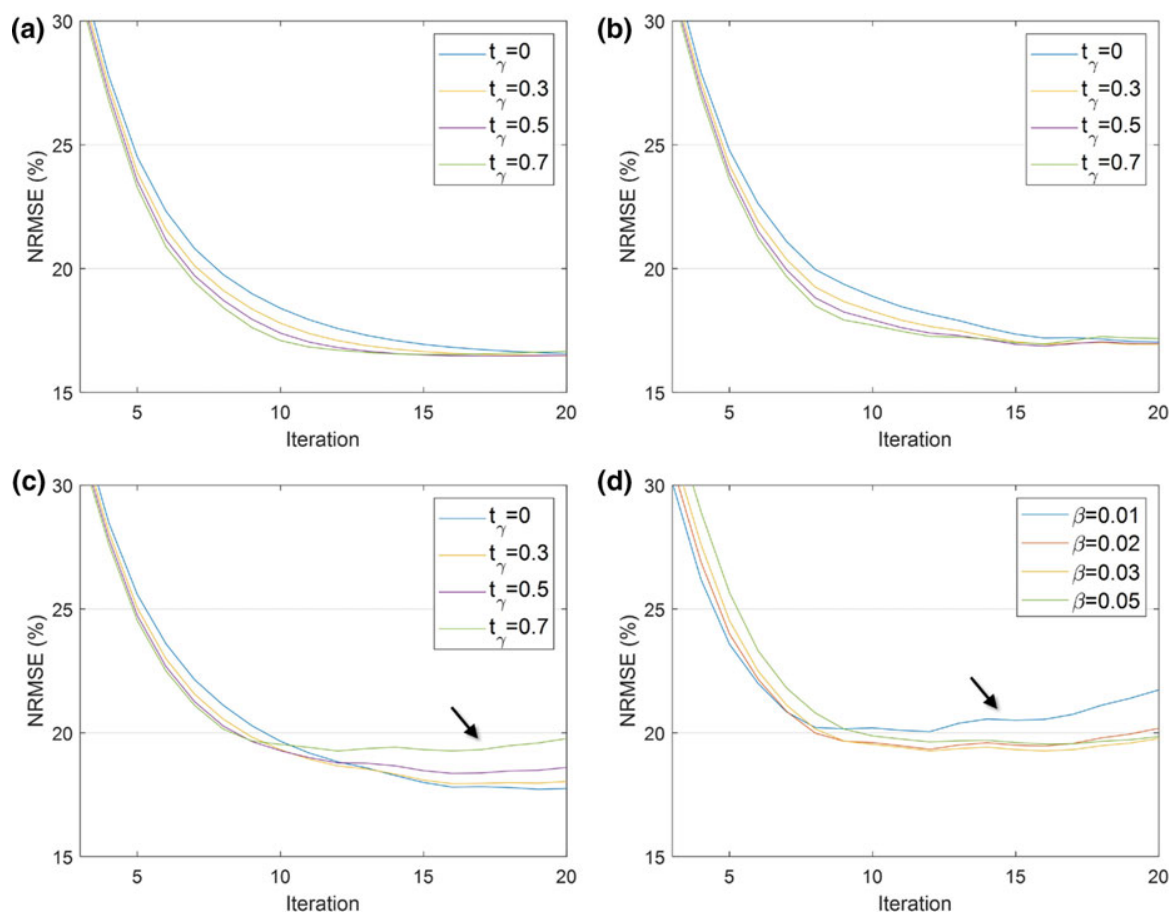

FIG. 13. Comparison of NRMSEs using different number of subsets of (a) 1 , (b) 8 , and (c) 16 combining with relaxation factors $\left(t_{\gamma}\right.$ ) of 0 (without momentum), 0.3 , 0.5 , and 0.7 , and (d) NRMSEs of $\beta=0.01,0.02,0.03$, and 0.05 with 16 subsets and relaxation factor of 0.7. [Color figure can be viewed at wileyonlinelibrary.com] 
combining with or without momentum were the highest for all ROIs. Here, SSIM considers mean (bias) and variance (noise). We confirmed that the mean values of hot regions of OS-EM, OS-NUSQS, and OS-NUSQS with momentum were almost identical. We additionally compared biases for a cold region in Fig. 10(b) (see arrow), where biases of OS-SQS, OS-EM, OS-NUSQS, and OS-NUSQS with momentum were $0.8 \%, 0.6 \%, 0.09 \%$, and $0.12 \%$. We confirmed that the OSNUSQS has faster convergence of lower SNR region compared to OS-EM.

Although the momentum method can increase the computing speed while preserving the uniform recovery, the reconstructed image can diverge when combined with OS; thus, the relaxation factor was incorporated into the momentum method. We performed an empirical comparison of relaxation factor, effect of subsets, and $\beta$, as similarly done by Berker et al. ${ }^{34}$ Figs. 13(a)-13(c) show NRMSE comparisons for various number of subsets of 1,8 , and 16 with relaxation factors $\left(t_{\gamma}\right)$ of 0 (without momentum), 0.3, 0.5, and 0.7, where the patient data and fixed $\beta=0.03$ were used. Without OS in Fig. 13(a), the OS-NUSQS with momentum decreased NRMSE monotonically with relaxation factors less than 0.7 . With 8 or 16 subsets, NRMSEs diverge with high relaxation factors [see arrows in Figs. 13(c) and 13(d)]. Figure 13(d) shows NRMSE comparison for various $\beta$ with 16 subsets and relaxation factor of 0.7 . In our observations, the proper

TABLE III. Computing time (s) of TOF forward and backward projectors without subsets using CPU and GPU. Specifically, 4-cores with $3.5 \mathrm{GHz}$ of a personal computer, 48-cores with $2.4 \mathrm{GHz}$ of a cluster server and Nvidia Geforce GTX 1080 for GPU implementation were compared. Computing time (s) of OS-EM, OS-NUSQS, and OS-NUSQS with momentum were compared. GPU time indicates one sub-iteration (one of eight subsets) computing time including TOF forward and backward projectors and quadratic penalty.

\begin{tabular}{lccc}
\hline \hline & Forward & Backward & Acceleration \\
\hline CPU(4) & 1920 & 2063 & $1.0 \times$ \\
CPU(48) & 368 & 480 & $5.0 \times$ \\
GPU & 25 & 25.1 & $80.0 \times$ \\
\hline \hline & OS-EM & OS-NUSQS & OS-NUSQS + mom \\
\hline GPU & 14.1 & 15.8 & 16.0 \\
\hline
\end{tabular}

hyperparameter and relaxation factor for 16 subsets were $\beta=0.05$ and $t_{\gamma}=0.5$, respectively. Similarly, the proper parameters for eight subsets were $\beta=0.03$ and $t_{\gamma}=0.5$. The results indicated that higher $\beta$ is required for higher relaxation factors to enable the convergence, however, different $\beta$ s converge to different solutions.

\section{C. Execution time}

In our iterative algorithms, the most time-consuming operations are forward and backward projectors. Table III compares the computing time using quad-core CPU with $3.5 \mathrm{GHz}$, 48-core CPU server with $2.4 \mathrm{GHz}$ and GPU (Geforce GTX 1080, Nvidia). Here, GE SIGNA TOF data without subset was used. For implementation, we used OpenMP for CPU and CUDA for GPU, specifically, 4-cores with $3.5 \mathrm{GHz}$ of a personal computer, 48-cores with $2.4 \mathrm{GHz}$ of a cluster server and Nvidia Geforce GTX 1080 were compared. We observed that the overall acceleration is about $80 \times$.

We also compared computing time (s) of OS-EM, OSNUSQS, and OS-NUSQS with momentum, which indicates one sub-iteration time of eight subsets including TOF forward and backward projectors and quadratic penalty. We observed that the OS-EM was $12 \%$ faster than the OS-NUSQS.

\section{DISCUSSION}

In Fig. 14(a), we observed variations of the NU factor ( $u$ in Eq. (27) by iterations. In early iterations, high-intensity regions have larger NU factors, indicating larger step size. After reaching similar recovery ratios, we observed that the step size in NU factor became more uniform, which means NU updates yield uniform recovery in early iterations. Figure 14(b) compares the Newton curvature $\left(\ddot{h}_{i}^{t}\left(\left[A_{t} x^{(n)}\right]_{i}\right)\right)$, approximate curvature $\left(1 / \max \left(\left[A_{t} x^{(n)}\right]_{i}, \epsilon\right)\right)$ and optimal curvature in Eq. (10). Although the optimal curvature is the fastest at early iterations, optimal curvature requires additional computations when $\left[A_{t} x^{(n)}\right]_{i}=0$. Because the NRMSE of optimal curvature became similar to NRMSEs of others after 10 iterations and the approximate curvature can be directly calculated by re-projected values in each iteration, we used the approximate
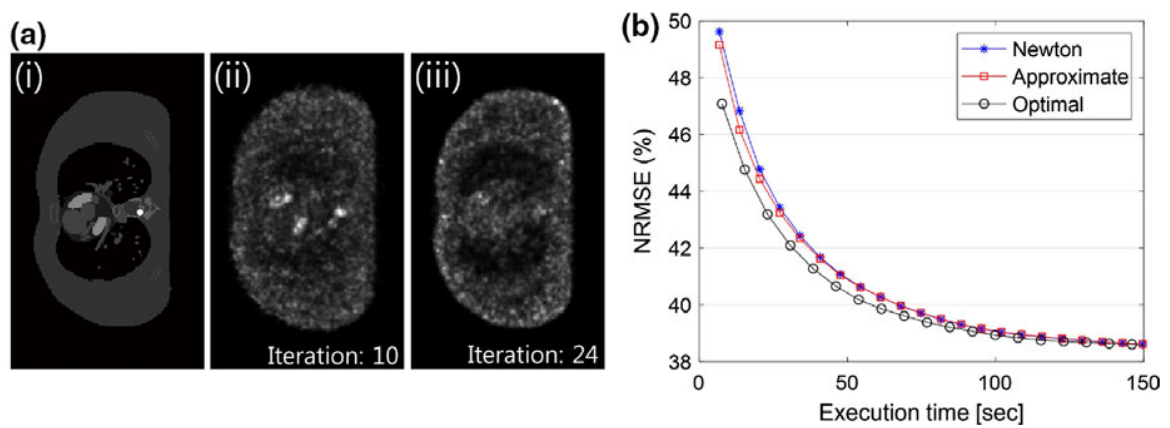

FIG. 14. (a)(i) Ground truth image and nonuniform factors at iterations of (ii) 10 and (iii) 24, and (b) comparison of NRMSEs of the reconstructed images over time using the Newton, approximate, and optimal curvatures. $\beta=0.2$ was used in simulation. [Color figure can be viewed at wileyonlinelibrary.com] 

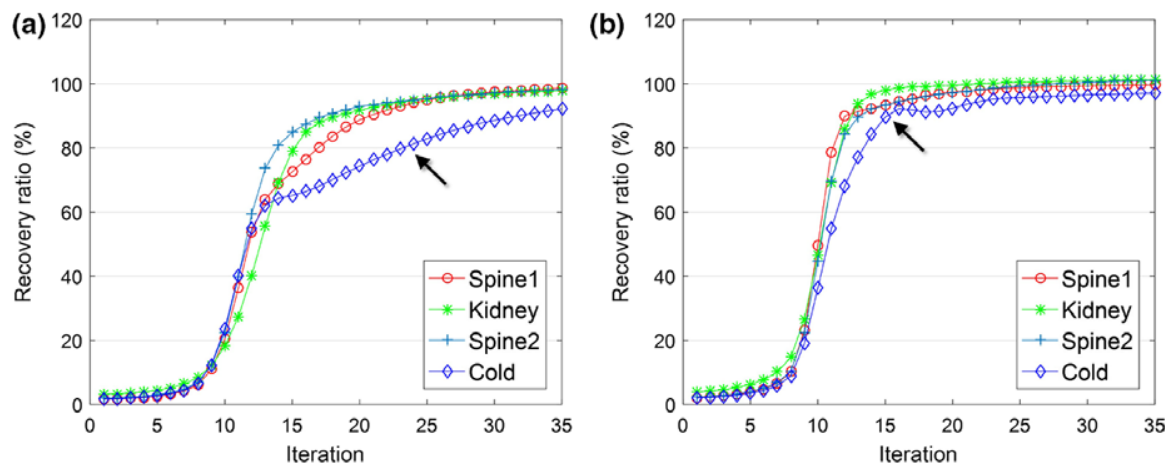

FIG. 15. Recovery ratio comparisons of (a) OS-EM and (b) OS-NUSQS. Here, the same initial image far from the ground truth was used in both (a) and (b). $\beta=0.03$ was used with patient data. [Color figure can be viewed at wileyonlinelibrary.com]

curvature in this paper. Note that although the approximate curvature does not hold inequalities of Eqs. (6), (12), (13) and (23) in general, we can reduce the computing time (13\% in our implementation).

In Figs. 7 and 11, the uniformity of recovery ratio was achieved at different number of iterations and we observed that the fast reaching of uniformity depends on the distance between the initial value and the converged value in reconstruction. We used 1 as an initial value for all voxels in both simulated data and real data in which the initial value of the cold region in the simulation was closer to the converged value and thus the uniformity was achieved faster. To investigate the impact of the initial value, particularly when it is very far from the solution, we compared recovery ratios of OS-EM and OS-NUSQS using different initial values in Fig. 15. We used a back-projected image as an initial image and same ROIs in Fig. 11 to compute the recovery ratio. Figure 15(a) showed that the cold region of OS-EM converged slower than hot regions. The uniformity of recovery ratios of OS-NUSQS was achieved around 15 iterations in Fig. 15(b) compared to 24 iterations in Fig. 11(c), which demonstrated that the fast reaching of uniformity depends on the initial value.

The momentum method can speed up convergence in early iterations particularly when it is combined with the ordered subset approach. However, because the gradient at each iteration is updated from a subset of whole data, the algorithm can diverge. Figure 13 demonstrated that the relaxation factor can achieve relatively faster convergence speed compared to the non-momentum method. The selection of the relaxation factor is still an open question. We empirically selected the optimal relaxation factor and the number of subsets, and will further investigate the relationship of optimal parameters and noise.

\section{CONCLUSIONS}

In conclusion, we derived an OS-NUSQS) with the Nesterov's momentum method for TOF PET reconstruction. The spatially NU step size in the proposed method provided uniform recovery ratios of different SNR regions. The computer simulation and clinical example showed that the proposed method converged uniformly regardless of hot and cold ROIs, and the contrast and SSIM were higher at early iterations using the proposed method than using the conventional OSEM and OS-SQS methods. Furthermore, our GPU implementation was able to achieve $80 \times$ acceleration compared to the implementation using 4-core CPU.

\section{ACKNOWLEDGMENT}

This research was supported by the National Institute of Health (NIH) P41 EB022544-6814 and NIH AG052653. The authors have no conflicts to disclose.

\footnotetext{
a) Authors to whom correspondence should be addressed Electronic mail: li.quanzheng@mgh.harvard.edu.
}

\section{REFERENCES}

1. Watson CC. An evaluation of image noise variance for time-of-flight PET. IEEE Trans Nucl Sci. 2007;54:1639-1647.

2. Conti M. State of the art and challenges of time-of-flight PET. Phys Med. 2009;25:1-11.

3. Snyder DL, Thomas LJ, Ter-Pogossian MM. A mathematical model for positron emission tomography systems having time-of-flight measurements. IEEE Trans Nucl Sci. 1981;3:3575-3583.

4. Tomitani T. Image reconstruction and noise evaluation in photon timeof-flight assisted positron emission tomography. IEEE Trans Nucl Sci. 1981;28:4581-4589.

5. Yamamoto M, Ficke DC, Ter-Pogossian MM. Experimental assessment of the gain achieved by the utilization of time-of-flight information in a positron emission tomograph (Super PETT I). IEEE Trans Med Imaging. 1982;1:187-192.

6. Budinger TF. Time-of-flight positron emission tomography: status relative to conventional PET. J Nucl Med. 1983;24:73-78.

7. Strother S, Casey M, Hoffman E. Measuring PET scanner sensitivity: relating countrates to image signal-to-noise ratios using noise equivalents counts. IEEE Trans Nucl Sci. 1990;37:783-788.

8. Conti M. Focus on time-of-flight PET: the benefits of improved time resolution. Eur J Nucl Med Mol Imaging. 2011;38:1147-1157.

9. Karp JS, Surti S, Daube-Witherspoon ME, Muehllehner G. Benefit of time-of-flight in PET: experimental and clinical results. J Nucl Med. 2008;49:462-470.

10. Hudson HM, Larkin RS. Accelerated image reconstruction using ordered subsets of projection data. IEEE Trans Med Imaging. 1994:13:601-609.

11. Ahn S, Fessler JA. Globally convergent image reconstruction for emission tomography using relaxed ordered sub-sets algorithms. IEEE Trans Med Imaging. 2003;22:613-626. 
12. Qi J, Leahy RM. A theoretical study of the contrast recovery and variance of MAP reconstructions from PET data. IEEE Trans Med Imaging. 1999;18:293-305.

13. Li Q, Bai B, Cho S, Smith A, Leahy R. Count independent resolution and its calibration. In: International Meeting on Fully Three-Dimensional Image Reconstruction in Radiology and Nuclear Medicine; 2009:223-226.

14. Cho JH, Fessler JA. Regularization designs for uniform spatial resolution and noise properties in statistical image reconstruction for 3-D Xray CT. IEEE Trans Med Imaging. 2015;34:678-689.

15. Yu Z, Thibault J, Bouman CA, Sauer KD, Hsieh J. Fast model-based Xray $\mathrm{CT}$ reconstruction using spatially non-homogeneous ICD optimization. IEEE Trans Image Process. 2011;20:161-175.

16. Van Slambrouck K, Nuyts J. Reconstruction scheme for accelerated maximum likelihood reconstruction: the patch-work structure. IEEE Trans Nucl Sci. 2014;61:173-181.

17. Kim D, Pal D, Thibault J-B, Fessler JA, Accelerating ordered subsets image reconstruction for X-ray CT using spatially nonuniform optimization transfer. IEEE Trans Med Imaging. 2013;32:1965-1978.

18. Nesterov Y. A method of solving a convex programming problem with convergence rate $O(1 / \mathrm{k} 2)$. In: Soviet Mathematics Doklady. Vol. 27. 1983:372-376.

19. Kim D, Ramani S, Fessler JA, Combining ordered subsets and momentum for accelerated X-ray CT image reconstruction. IEEE Trans Med Imaging. 2015;34:167-178

20. Segars W, Mahesh M, Beck T, Frey E, Tsui B. Realistic CT simulation using the 4D XCAT phantom. Med Phys. 2008;35:3800-3808.

21. Kim K, Wu D, Gong K, et al. Penalized PET Reconstruction Using Deep Learning Prior and Local Linear Fitting. IEEE Trans Med Imaging. 2018;37:1478-1487.

22. Humm JL, Rosenfeld A, Del Guerra A. From PET detectors to PET scanners. Eur J Nucl Med Mol Imaging. 2003;30:1574-1597.

23. Vandenberghe S, Karp J. Rebinning and reconstruction techniques for 3D TOF-PET. Nucl Instrum Methods Phys Res Sect A 2006;569: $421-424$.
24. Qi J, Leahy RM, Cherry SR, Chatziioannou A, Farquhar TH. High-resolution 3D Bayesian image reconstruction using the microPET small-animal scanner. Phys Med Biol. 1998;43:1001-1013.

25. Fessler JA, Erdogan H. A paraboloidal surrogates algorithm for convergent penalized-likelihood emission image reconstruction. In: Nuclear Science Symposium, Vol. 2. IEEE; 1998:1132-1135.

26. Erdogan H, Fessler JA. Ordered subsets algorithms for transmission tomography. Phys Med Biol. 1999;44:2835-2851.

27. Kim D, Fessler JA. Parallelizable algorithms for X-ray CT image reconstruction with spatially non-uniform updates. The Second International Conference on Image Formation in X-ray Computed Tomography; 2012:33-36.

28. De Pierro AR. A modified expectation maximization algorithm for penalized likelihood estimation in emission tomography. IEEE Trans Med Imaging. 1994;14:132-137.

29. Pratx G, Chinn G, Habte F, Olcott P, Levin C. Fully 3-D list-mode OSEM accelerated by graphics processing units. In: Nuclear Science Symposium and Medical Imaging Conference, Vol. 4. IEEE; 2006:2196-2202.

30. Bai B, Smith A. Fast 3D iterative reconstruction of PET images using PC graphics hardware. In: IEEE Nuclear Science Symposium and Medical Imaging Conference, Vol. 5. IEEE; 2006:2787-2790

31. Kim K, Ye J. Fully 3D iterative scatter-corrected OSEM for HRRT PET using a GPU. Phys Med Biol. 2011;56:4991-5009.

32. Kim K, Son YD, Bresler Y, Cho ZH, Ra JB, Ye JC. Dynamic PET reconstruction using temporal patch-based low rank penalty for ROIbased brain kinetic analysis. Phys Med Biol. 2015;60:2019-2046.

33. Cui J-Y, Pratx G, Prevrhal S, Levin CS. Fully 3D list-mode time-offlight PET image reconstruction on GPUs using CUDA. Med Phys. 2011;38:6775-6786.

34. Berker Y, Karp JS, Schulz V. Numerical algorithms for scatter-toattenuation reconstruction in PET: Empirical comparison of convergence, acceleration, and the effect of subsets. IEEE Trans Radiat Plasma Med Sci. 2017;1:426-434. 